\title{
Parallel Projected Sphere on a Plane: A New Plane-Geometric Investigation
}

\author{
Thomas L. Toulias* and George E. Lefkaditis \\ (Communicated by Levent Kula)
}

\begin{abstract}
Consider three concentric ellipses $E_{i}, i=1,2,3$, each defined by a pair of conjugate semi-diameters taken from a given bundle of three coplanar line segments (where only two of them are permitted to coincide). In a proof by G. A. Peschka of the Karl Pohlke's Fundamental Theorem of Axonometry, a parallel projection of a sphere onto a plane, say $\mathbb{E}$, is adopted to show that a new concentric (to $E_{i}$ ) ellipse $E$ exists, "circumscribing" all $E_{i}$, i.e. $E$ is simultaneously tangent to all $E_{i} \subset \mathbb{E}, i=1,2,3$. Motivated by the above, this paper investigates the plane-geometric problem of determining all the existing circumscribing ellipses (like $E$ ) of $E_{i}, i=1,2,3$, exclusively from the Analytic Plane Geometry's point of view (unlike the sphere's parallel projection that requires the adoption of a three-dimensional space). It is proved that, at most, two circumscribing ellipses (of $E_{i}$ ) exist. One of them is always existing while, under certain conditions, another circumscribing ellipse (of $E_{i}$ ), say $E^{*}(\neq E)$, can also exist. Moreover, in case this second circumscribing ellipse $E^{*}$ does not exist, then a hyperbola (concentric to $E_{i}$ ) exists instead, and is (simultaneously) tangent to all $E_{i}, i=1,2,3$. The above results and their calculations are demonstrated by various examples and figures.
\end{abstract}

Keywords: Pohlke's Theorem; conjugate semi-diameters of an ellipse; affine transformations; orthogonal decomposition of a symmetric matrix. AMS Subject Classification (2010): Primary: 51N10; Secondary: $15 A 18$.

\section{Introduction}

This work is motivated by a work of Müller and Kruppa [4], where they suggested a proof (out of many in the literature) of Karl Pohlke's Theorem, also known as the Axonometry's Fundamental Theorem. Recalling Pohlke's Theorem, we consider a bundle of three arbitrary chosen coplanar line segments, say $O P, O Q$ and $O R$, where only one of them can be of zero length (non-degenerated segments). Under the assumption that the points $O, P, Q, R \in \mathbb{E}$ are not collinear (belonging to a plane $\mathbb{E}$ ), their corresponding line segments, as above, can always be considered as parallel projections of three other line segments of the three-dimensional space, say $O^{*} P^{*}, O^{*} Q^{*}$ and $O^{*} R^{*}$ respectively, having equal length and being orthogonal with each other; see Fig. 1 . The orthogonal projection can also be considered as a special case of the above parallel projection. For further reading about Pohlke's Theorem see also [1] and [6].

The specific method in [4] of the proof of Pohlke's Theorem (which consists of finding the orthogonal segments $O^{*} P^{*}, O^{*} Q^{*}$ and $\left.O^{*} R^{*}\right)$ is based on the adoption of a parallel projection, say $\mathscr{P}$, applied on an appropriate sphere $S$ onto plane $\mathbb{E}$ (on which $O P, O Q$ and $O R$ lie). The following property of three concentric ellipses (proven through the use of $\mathscr{P}$ ) is stated here as a Proposition, [4, pg. 244]:

Proposition 1.1. Consider four non-collinear points $O, P, Q, R$ on a plane, forming a bundle of three line segments $O P, O Q$ and $O R$, where only two of them are permitted to coincide. If the pairs $(O P, O R),(O Q, O R)$ and $(O P, O Q)$ are considered to be pairs of conjugate semi-diameters defining the ellipses, say $E_{1}, E_{2}$ and $E_{3}$ respectively, then a new ellipse $E$ (concentric to $E_{i}$ ) exists and is tangent to all $E_{i}, i=1,2,3$.

The use of sphere, for the proving of the above, appears for the first time in a work by J. W. v. Deschwanden and subsequently by G. A. Peschka in his elementary proof of Pohlke's Fundamental Theorem of Axonometry; 


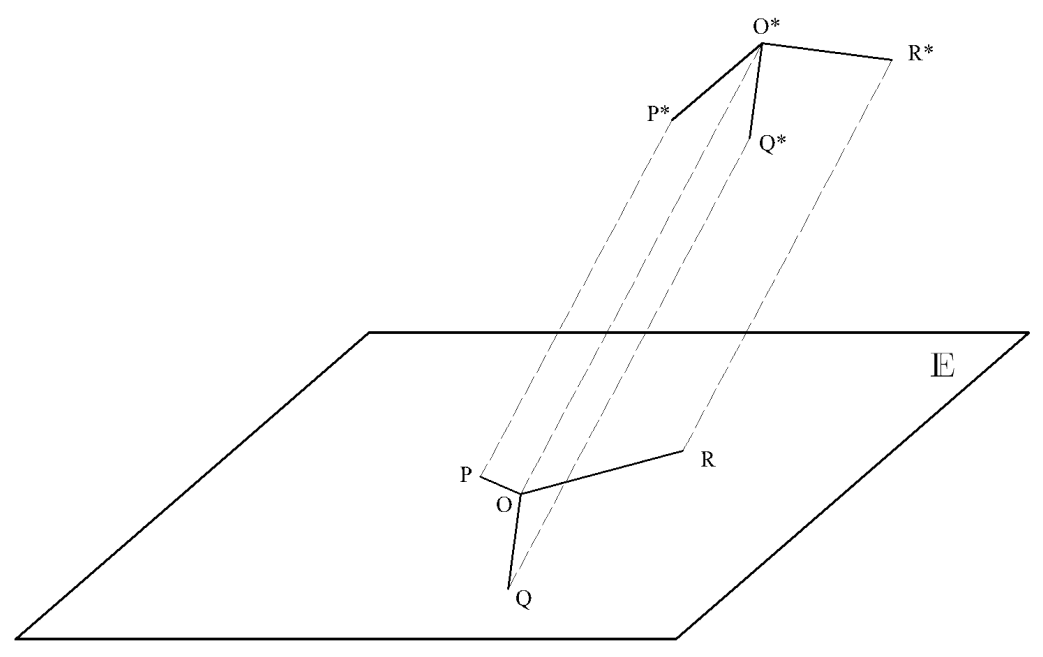

Figure 1. Parallel projection for the Pohlke's Theorem.

see [5]. Under this parallel projection $\mathscr{P}$, a cylindrical surface is created, tangent to sphere $S$ and around its maximum circle, say $k \subset S$; see Fig. 2. Hence, $k$ is the contour of sphere $S$ through $\mathscr{P}$, and it is parallel-projected (via $\mathscr{P}$ ) onto an ellipse $E$ of plane $\mathbb{E}$, while the orthogonal line segments $O^{*} P^{*}, O^{*} Q^{*}$ and $O^{*} R^{*}$ are parallelprojected onto radii $O P, O Q$ and $O R$ of the ellipses $E_{i}, i=1,2,3$. Therefore $E$ is circumscribing all $E_{i}, i=1,2,3$. According to proof of Proposition 1.1, these ellipses are defined by three pairs of conjugate semi-diameters, or conjugate radii, $(O P, O R),(O Q, O R)$ and $(O P, O Q)$ respectively, which are parallel projections, through $\mathscr{P}$, of the corresponding three maximum circles on sphere $S$. These maximum circles belong to planes (perpendicular to each other) spanned, respectively, by $\left\{O^{*} P^{*}, O^{*} R^{*}\right\},\left\{O^{*} Q^{*}, O^{*} R^{*}\right\}$ and $\left\{O^{*} P^{*}, O^{*} Q^{*}\right\}$. Figure 2 demonstrates the above projections (used by G. A. Peschka) for the proof of Proposition 1.1.

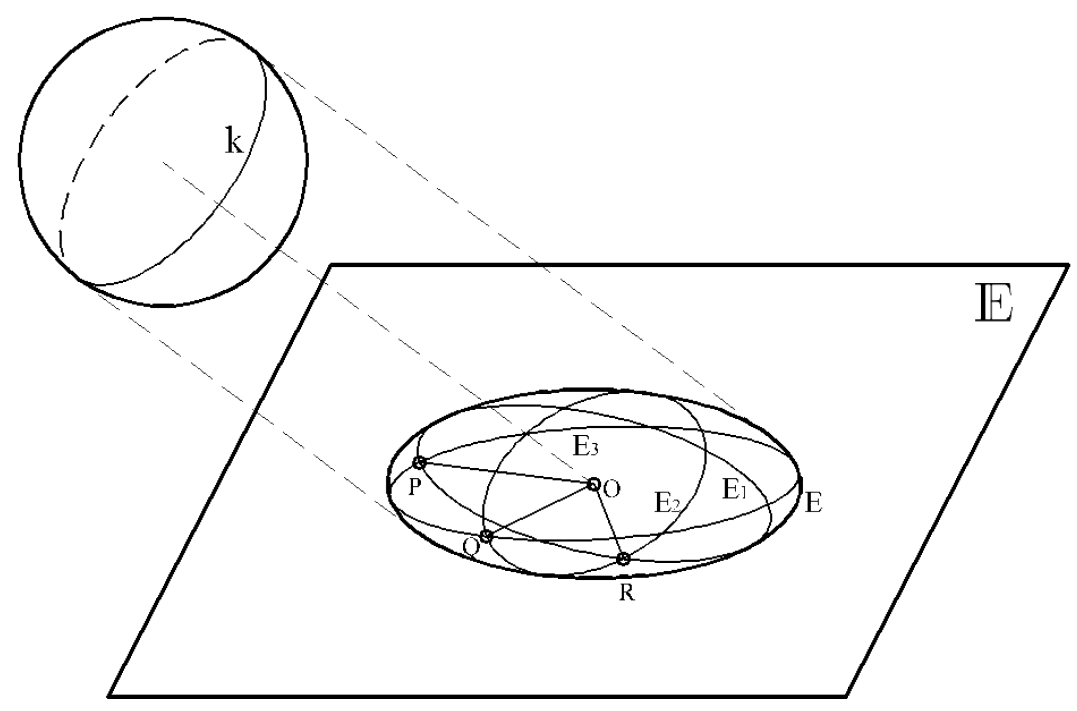

Figure 2. Pohlke's Theorem through a parallel-projected sphere onto plane $\mathbb{E}$.

One can notice that the problem in Proposition 1.1 is, by its nature, a plane-geometric (two-dimensional) problem. Moreover, the question of how many common tangent ellipses circumscribing all $E_{i}$ (like $E$ ) exist, is open. The problem, of finding the $E_{i}{ }^{\prime} \mathrm{s}$ "circumscribing" ellipse $E$ is the problem of finding a "common tangential ellipse" (c.t.e.) $E$ of all the ellipses $E_{i}, i=1,2,3$. A visualization of a c.t.e. is given in Fig. 3. For the topic of concentric circumscribing (tangent) ellipses see [2] among others.

The present paper, in particular, provides a thorough investigation of the the two-dimensional problem of finding a c.t.e. of three given concentric ellipses is addressed, where each of these three ellipses is defined by 


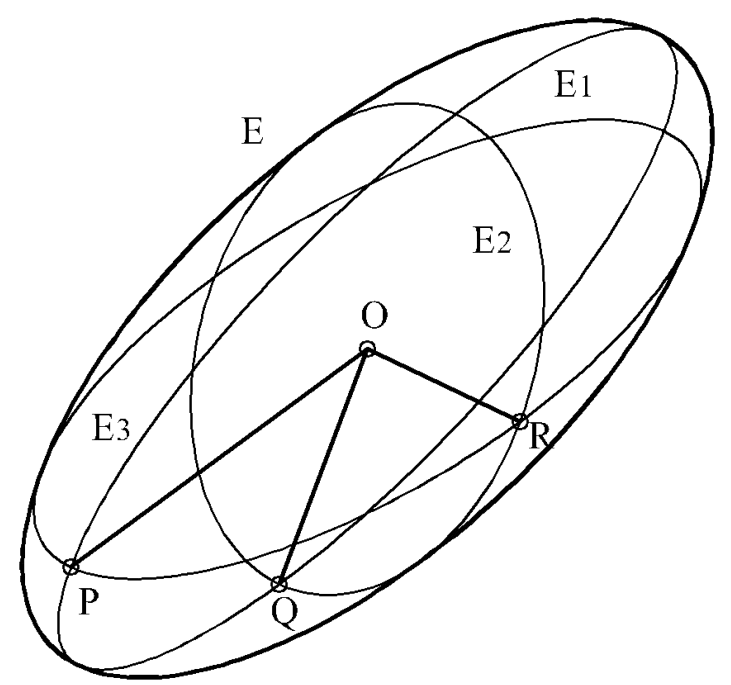

Figure 3. A common tangential ellipse $E$ of all $E_{i}, i=1,2,3$.

two conjugate radii which belong to a given bundle of three (coplanar) non-degenerated line segments (where only two of them can coincide). Useful formulas are also provided and various examples demonstrate the results. Note that Lefkaditis et al. in [3] proposed a construction method of an enveloping ellipse $E$ utilizing Synthetic Projective Geometry of the Plane. Furthermore, we present also a method which provides (not some but) all the existing common tangential ellipses of $E_{i}, i=1,2,3$. In fact, it is proved that at, most, two common tangential ellipses can exist. One of them always exists (as it is also expected from the sphere's parallel projection $\mathscr{P})$ while, under certain assumptions, one more c.t.e., say $E^{*}(\neq E)$, can also exist. It is worth mentioning that, when there is only one c.t.e. of $E_{i}$ (meaning that a second c.t.e. ellipse $E^{*}$ does not exists), then a common tangential hyperbola exists instead, i.e. a hyperbola (concentric to $E$ and $E_{i}$ ) which is (simultaneously) tangent to all $E_{i}, i=1,2,3$.

Affine transformations play a key role in this investigation. A study of certain affine transformations is carried out in Section 2, that helps simplify the corresponding formulations. In Section 3, these transformations first apply for the special case where one of the ellipses $E_{i}$ is assumed to be a circle, and then the investigation is generalized for the case where all $E_{i}$ are ellipses in general.

\section{Ellipse as an Affinic Circle}

In this section, a certain affine transformation is studied transforming circles into ellipses, which is needed for the development of our study in Section 3. Firstly, we consider the following preliminary Lemma concerning the rotation of an ellipse around its center.

Lemma 2.1. Let $E$ be an ellipse with principal radii $0<b<a$, centered at the origin $O$ of an orthonormal coordinate system. The analytical expression of the rotated ellipse $E_{\theta}$ by an angle $\theta \in[-\pi, \pi]$ around its center $O$, is then given by

$$
E_{\theta}:\left(a^{2} \sin ^{2} \theta+b^{2} \cos ^{2} \theta\right) x^{2}-\left(a^{2}-b^{2}\right)(\sin 2 \theta) x y+\left(a^{2} \cos ^{2} \theta+b^{2} \sin ^{2} \theta\right) y^{2}=a^{2} b^{2} .
$$

Proof. Consider the canonical form of $E:(x / a)^{2}+(y / b)^{2}=1$. Then, the result is obtained through the affine transformation (rotation) $R_{\theta}$ defined by $\mathbf{x}^{\prime}=R_{\theta}(\mathbf{x}):=\mathbf{R}_{\theta} \mathbf{x}$, where $\mathbf{x}:=(x, y)^{\mathrm{T}} \in \mathbb{R}^{2 \times 1}$ and $\mathbf{x}^{\prime}:=\left(x^{\prime}, y^{\prime}\right)^{\mathrm{T}} \in \mathbb{R}^{2 \times 1}$ denote the coordinates' vectors of points $X$ and $X^{\prime}$, while

$$
\mathbf{R}_{\theta}:=\left(\begin{array}{rr}
\cos \theta & -\sin \theta \\
\sin \theta & \cos \theta
\end{array}\right) \in \mathbb{R}_{\perp}^{2 \times 2}
$$

is the usual rotation (orthonormal) matrix which defines the rotation $X^{\prime}:=R_{\theta}(X)$ of the Euclidean plane $\mathbb{R}^{2}$ by an angle $\theta$ around its origin $O$. Thus, applying $R_{\theta}$ to the matrix form of ellipse $E$, i.e. $E: \mathbf{a x}^{\mathrm{T}}=1, \mathbf{a}:=(a, b) \in \mathbb{R}^{2}$, we obtain the rotated ellipse $E_{\theta}:=R_{\theta}(E)$, expressed by (2.1). 
A given circle can be transformed into an ellipse through an axis-invariant affine transformation which shall be called "axis shear". Specifically, we shall call " $x$-shear" the axis shear that preserves the horizontal axis $x^{\prime} O x$ of an given coordinate system (c.s.), while " $y$-shear" shall be called the axis shear which preserves the corresponding c.s.'s vertical axis $y^{\prime} O y$. Hence, the $y$-shear transforms the orthonormal vector base $\mathscr{B}=\left\{\mathbf{e}_{1}, \mathbf{e}_{2}\right\}$ into $\mathscr{B}^{\prime}=\left\{\mathbf{u}, \mathbf{e}_{2}\right\}$, while the $x$-shear transforms the vector base $\mathscr{B}$ into $B^{\prime}=\left\{\mathbf{e}_{1}, \mathbf{u}\right\}, \mathbf{u} \in \mathbb{R}^{2 \times 1} \backslash\{\mathbf{0}\}$, where $\mathbf{e}_{1}$ and $\mathbf{e}_{2}$ denote the usual orthonormal vectors $\mathbf{e}_{1}:=(1,0)^{\mathrm{T}}$ and $\mathbf{e}_{2}:=(0,1)^{\mathrm{T}}$ of $\mathbb{R}^{2}$. Note that the c.s. spanned by the vector base $\mathscr{B}$ is an orthonormal c.s. (o.s.c.). The vector $\mathbf{u} \neq \mathbf{0}$ shall be called as the "shearing vector", while the $\mathbf{u}^{\prime}$ s angle (with respect to the adopted o.c.s.) $\omega:=\measuredangle\left(\mathbf{e}_{1}, \mathbf{u}\right) \in(-\pi, \pi)$ shall often be called as the "shearing angle". In particular, the $x$-shear, say $S_{x}$, is an affine transformation defined through its matrix representation $\mathbf{x}^{\prime}=S_{x}(\mathbf{x}):=\mathbf{N}_{x} \mathbf{x}$ where the transformation matrix $\mathbf{N}_{x}$ is given by

$$
\mathbf{N}_{x}=\left(\mathbf{e}_{1}, \mathbf{u}\right):=\left(\begin{array}{ll}
1 & u \cos \omega \\
0 & u \sin \omega
\end{array}\right)
$$

with $\omega \in(-\pi, \pi)$ being the shearing angle of the $x$-shear $S_{x}$. Respectively, the $y$-shear, say $S_{y}$, is defined by $\mathbf{x}^{\prime}=S_{y}(\mathbf{x}):=\mathbf{N}_{y} \mathbf{x}$, where the transformation matrix $\mathbf{N}_{y}$ is given by

$$
\mathbf{N}_{y}=\left(\mathbf{u}, \mathbf{e}_{2}\right):=\left(\begin{array}{ll}
u \cos \omega & 0 \\
u \sin \omega & 1
\end{array}\right)
$$

with $\omega \in(-\pi, \pi)$ being the corresponding $S_{y}$ 's shearing angle, which is the same as in (2.3) (as $S_{y}$ is referring to the same sharing vector $\mathbf{u}$ as $S_{x}$ ). The $x$-, or $y$-sears, are therefore completely defined through a given shearing vector $\mathbf{u} \in \mathbb{R}^{2 \times 1}$, i.e. by its length $u \in \mathbb{R}_{+}$, and its angle $\omega \in(-\pi, \pi)$ (with respect to some c.s.).

For the ellipse derived from a $y$-shear of a circle we consider the following. Note that with the term "directive angle" of an ellipse we shall refer hereafter to the angle formed by the ellipse's major axis with respect to the horizontal $x^{\prime} O x$ axis of an adopted c.s. In general, the directive angle of an ellipse with respect to a given line shall refer to the angle between the ellipse's major axis and this given line.

Lemma 2.2. Let $C$ be a circle of radius $\rho>0$ centered at the origin $O$ (of an o.c.s.). The $y$-sheared circle $S_{y}(C)$ corresponds to an ellipse, centered also at the origin $O$, with analytical expression

$$
S_{y}(C):\left(1+u^{2} \sin ^{2} \omega\right) x^{2}-u^{2}(\sin 2 \omega) x y+u^{2}\left(\cos ^{2} \omega\right) y^{2}=\rho^{2} u^{2} \cos ^{2} \omega .
$$

where $u>0$ and $\omega \in[-\pi, \pi]$ are the corresponding $S_{y}$ 's shearing vector length and angle. The principal radii $0<b<a$ of $S_{y}(C)$ are given by

$$
a, b=\frac{\sqrt{2} \rho u|\cos \omega|}{\sqrt{u^{2}+1 \pm \sqrt{u^{4}+1-2 u^{2} \cos 2 \omega}}},
$$

where the minus sign corresponds to the major radius a while the plus sign to the minor radius $b$. Note that the surface area of $S_{y}(C)$ adopts the compact form $A=\pi u \rho^{2}|\cos \omega|$. Moreover, it holds that $|\omega|<|\theta|$ where $\theta$ is the directive angle of the ellipse $S_{y}(C)$, which is given through the $S_{y}(C)$ 's major axis slope $\tan \theta$, by

$$
\tan \theta=\frac{u^{2} \sin 2 \omega}{u^{2} \cos 2 \omega-1+\sqrt{u^{4}+1-2 u^{2} \cos 2 \omega}} .
$$

Proof. For the $y$-shear $S_{y}$, defined by $\mathbf{x}^{\prime}=S_{y}(\mathbf{x}):=\mathbf{N}_{y} \mathbf{x}$, with transformation matrix $\mathbf{N}_{y}$ as in (2.4), we have that $\mathbf{x}=S_{y}^{-1}\left(\mathbf{x}^{\prime}\right)=\mathbf{N}_{y}^{-1} \mathbf{x}^{\prime}$, i.e.

$$
\left(\begin{array}{l}
x \\
y
\end{array}\right)=: \mathbf{x}=\mathbf{N}_{y}^{-1} \mathbf{x}^{\prime}=\left(\begin{array}{cc}
u \cos \omega)^{-1} & 0 \\
-\tan \omega & 1
\end{array}\right)\left(\begin{array}{l}
x^{\prime} \\
y^{\prime}
\end{array}\right)=\left(\begin{array}{c}
\frac{x^{\prime}}{u \cos \omega} \\
y^{\prime}-x^{\prime} \tan \omega
\end{array}\right),
$$

and thus the matrix representation form $C: \mathbf{x}^{\mathrm{T}} \mathbf{x}=\rho^{2}$ of the circle $C: x^{2}+y^{2}=\rho^{2}$ implies that $S_{y}(C)$ : $\mathbf{x}^{\prime \mathrm{T}}\left(\mathbf{N}_{y}^{-1}\right)^{\mathrm{T}} \mathbf{N}_{y}^{-1} \mathbf{x}^{\prime}=\rho^{2}$ or, equivalently, $S_{y}(C): \mathbf{x}^{\mathrm{T}}\left(\mathbf{N}_{y}^{-1}\right)^{\mathrm{T}} \mathbf{N}_{y}^{-1} \mathbf{x}=\rho^{2} \quad\left(\right.$ as $S_{y}(C)$ is referred again to the adopted o.c.s.). Therefore, $S_{y}(C): x^{2}+u^{2}(y \cos \omega-x \sin \omega)^{2}=\rho^{2} u^{2} \cos ^{2} \omega$, and hence (2.5) is obtained. The ellipse $S_{y}(C)$ is centered also at the origin $O$. This is true because $x$ - and $y$-shears preserve the center $O$ as it belongs both to the preserved coordinates' axes $x^{\prime} O x$ and $y^{\prime} O y$.

In order to obtain the principal radii $0<b<a$, the ellipse $S_{y}(C)$ has to be rotated by its directive angle, say $\theta$, around its center $O$ until its canonical form is obtained (on the adopted o.c.s.), i.e. until $S_{y}(C)$ is transformed 
into $E_{y}: \widetilde{\mathbf{x}}^{\mathrm{T}} \widetilde{\mathbf{D}} \widetilde{\mathbf{x}}=1, \widetilde{\mathbf{x}} \in \mathbb{R}^{2 \times 1}$, where $\widetilde{\mathbf{D}} \in \mathbb{R}_{\text {diag }}^{2 \times 2}$ is a real diagonal $2 \times 2$ matrix. For this purpose, we consider the rotation transform

$$
\widetilde{\mathbf{x}}=R_{\theta}(\mathbf{x}):=\mathbf{R}_{\theta} \mathbf{x}, \quad \mathbf{x} \in \mathbb{R}^{2 \times 1},
$$

with rotation matrix $\mathbf{R}_{\theta} \in \mathbb{R}_{\perp}^{2 \times 2}$ as in (2.2), that provides the ellipse $S_{y}(C)$, as in (2.5), from its requested canonical form $E_{y}: \widetilde{\mathbf{x}}^{\mathrm{T}} \widetilde{\mathbf{D}} \widetilde{\mathbf{x}}=1$, i.e. $R_{\theta}\left(E_{y}\right)=S_{y}(C)$ or $E_{y}=R_{\theta}^{-1}\left(S_{y}(C)\right)$. Recall the matrix representation of the ellipse $S_{y}(C)$ (mentioned earlier), i.e.

$$
S_{y}(C): \mathbf{x}^{\mathrm{T}} \mathbf{N} \mathbf{x}=\rho^{2}, \quad \mathbf{N}:=\left(\mathbf{N}_{y}^{-1}\right)^{\mathrm{T}} \mathbf{N}_{y}^{-1} \in \mathbb{R}_{\mathrm{sym}}^{2 \times 2} .
$$

Then, (2.9) implies that $\mathbf{x}=\mathbf{R}_{\theta}^{-1} \mathbf{x}=\mathbf{R}_{\theta}^{\mathrm{T}} \widetilde{\mathbf{x}}=\mathbf{R}_{-\theta} \widetilde{\mathbf{x}}$, and by substitution to (2.10), it holds that

$$
E_{y}=R_{\theta}^{-1}\left(S_{y}(C)\right)=\left(R_{\theta}^{-1} \circ S_{y}\right)(C): \widetilde{\mathbf{x}}^{\mathrm{T}} \mathbf{R}_{-\theta} \mathbf{N} \mathbf{R}_{\theta} \widetilde{\mathbf{x}}=\rho^{2} .
$$

Recall that the inverse of an orthogonal matrix equals to the transpose of the matrix, i.e. $\mathbf{M}^{-1}=\mathbf{M}^{\mathrm{T}}$ for $\mathbf{M} \in \mathbb{R}_{\perp}^{n \times n}$, $n \in \mathbb{N}$. By orthogonal decomposition of the symmetric matrix $\mathbf{N} \in \mathbb{R}_{\text {sym }}^{2 \times 2}$ we obtain that $\mathbf{N}=\mathbf{V D V}^{\mathrm{T}}$, where $\mathbf{D}:=\operatorname{diag}\left(d_{1}, d_{2}\right) \in \mathbb{R}_{\text {diag }}^{2 \times 2}$ is the spectral matrix of $\mathbf{N}$ (i.e. the diagonal $2 \times 2$ matrix of the eigenvalues $d_{1}$ and $d_{2}$ of $\left.\mathbf{N}\right)$ and $\mathbf{V} \in \mathbb{R}_{\perp}^{2 \times 2}$ is the orthonormal $2 \times 2$ matrix of the eigenvectors corresponding to eigenvalues $d_{1}$ and $d_{2}$. Thus, $\mathbf{D}=\mathbf{V}^{\mathrm{T}} \mathbf{N V}$, and by setting $\mathbf{R}_{\theta}:=\mathbf{V}$ (i.e. we adopt as orthonormal rotation matrix $\mathbf{R}_{\theta}$ the (orthonormal) eigenvalues matrix $\mathbf{V})$, the relation (2.11) implies the canonical form of $S_{y}(C)$, i.e. $E_{y}: \widetilde{\mathbf{x}}^{\mathrm{T}}\left(\rho^{-2} \mathbf{D}\right) \widetilde{\mathbf{x}}=1$. Hence, the major and minor radius of the ellipse $E_{y}:\left(d_{1} \tilde{x} / \rho\right)^{2}+\left(d_{2} \tilde{y} / \rho\right)^{2}=1$ (which are the same for the rotated ellipse $\left.S_{y}(C)\right)$ are then given by $a=\rho / \sqrt{\max \left\{d_{1}, d_{2}\right\}}$ and $b=\rho / \sqrt{\min \left\{d_{1}, d_{2}\right\}}$ respectively.

The eigenvalues $d_{1}$ and $d_{2}$ of the symmetric matrix $\mathbf{N}$ can be calculated through the roots of the $\mathbf{N}^{\prime} \mathrm{s}$ characteristic polynomial $P_{\mathbf{N}}(d):=\left|\mathbf{N}-d \mathbb{I}_{2}\right|, d \in \mathbb{R}, i=1,2$, where $\mathbb{I}_{2}$ being the unitary $2 \times 2$ matrix. After some algebra, we derive that

$$
d_{i}=\frac{\sec ^{2} \omega}{2 u^{2}}\left[\left(u^{2}+1\right)+(-1)^{i-1} \sqrt{u^{4}+1-2 u^{2} \cos 2 \omega}\right], \quad i=1,2,
$$

with $0<\left(u^{2}-1\right)^{2}=u^{4}-2 u^{2}+1<u^{4}+1-2 u^{2} \cos 2 \omega$ for every $u>0$, and therefore, the requested major and minor radius of the ellipse $S_{y}(C)$ correspond to the eigenvalues $d_{2}$ and $d_{1}$ respectively (as $d_{1}>d_{2}$ ), and hence given by (2.6).

Moreover, the non-unitary orthogonal eigenvectors $\mathbf{v}_{i}=\left(v_{i ; 1}, v_{i ; 2}\right)^{\mathrm{T}} \in \mathbb{R}^{2 \times 1}$ that correspond to its eigenvalues $d_{i}, i=1,2$, i.e. $\mathbf{V}=\left(\mathbf{v}_{1}, \mathbf{v}_{2}\right) \in \mathbb{R}_{\perp}^{2 \times 2}$, are then calculated through (2.12) and (after some algebra), are found to be

$$
\mathbf{v}_{i}=\left(-\frac{u^{2}+1-2 u^{2} \cos ^{2} \omega+(-1)^{i-1} \sqrt{u^{4}+1-2 u^{2} \cos 2 \omega}}{u^{2} \sin 2 \omega}, 1\right)^{\mathrm{T}}, \quad i=1,2 .
$$

Hence, as the major radius $a$ corresponds to the eigenvalue $d_{2}$ (shown earlier), the directive angle $\theta$ of the ellipse $S_{y}(C)$ is then of the form $\theta=\angle\left(\mathbf{e}_{1}, \mathbf{v}_{2}\right)=\arctan \left(v_{2 ; 2} / v_{2 ; 1}\right)$ and it is given by (2.7).

Notice also the fact that the assumption $\tan \theta<\tan \omega$ yields, through (2.7), that $u^{2}+1>\sqrt{u^{4}+1-2 u^{2} \cos 2 \omega}$, for $\omega>0$, which cannot hold (as it would then implies that $\cos ^{2} \omega<0$ ). Therefore, it holds that $\tan \theta>\tan \omega$ when $\omega>0$ while, similarly, $\tan \theta<\tan \omega$ when $\omega<0$. Hence $|\theta|>|\omega|$.

Working similarly for the $x$-shear of the circle $C$, the following holds.

Lemma 2.3. Let $C$ be a circle of radius $\rho>0$ centered at the origin $O$ (of an o.c.s.). The $x$-sheared circle $S_{x}(C)$ corresponds to an ellipse, centered also at the origin $O$, with analytical expression

$$
S_{x}(C): u^{2}\left(\sin ^{2} \omega\right) x^{2}-u^{2}(\sin 2 \omega) x y+\left(1+u^{2} \cos ^{2} \omega\right) y^{2}=\rho^{2} u^{2} \sin ^{2} \omega .
$$

where $u>0$ and $\omega \in[-\pi, \pi]$ are the corresponding shearing vector's length and angle. The principal radii $0<b<a$ of $S_{x}(C)$ are given by

$$
a, b=\frac{\sqrt{2} \rho u|\sin \omega|}{\sqrt{u^{2}+1 \pm \sqrt{u^{4}+1+2 u^{2} \cos 2 \omega}}},
$$

where the minus sign corresponds to the major radius a while the plus sign to the minor radius $b$. Note that the surface area of $S_{x}(C)$ adopts the compact form $A=\pi u \rho^{2}|\sin \omega|$. Moreover, it holds that $|\omega|>|\theta|$ where $\theta$ is the directive angle of the ellipse $S_{x}(C)$, which is given, through the $S_{x}(C)$ 's major axis slope $\tan \theta$, by

$$
\tan \theta=\frac{u^{2} \sin 2 \omega}{1+u^{2} \cos 2 \omega+\sqrt{u^{4}+1+2 u^{2} \cos 2 \omega}} .
$$


Proof. Similarly to the proof of Lemma 2.2, the $x$-shear transformation $S_{x}$, is defined by the matrix relation $\mathbf{x}^{\prime}=S_{x}(\mathbf{x}):=\mathbf{N}_{x} \mathbf{x}$ with transformation matrix $\mathbf{N}_{x}$ as in (2.3). Thus, we obtain $S_{x}(C): \mathbf{x}^{\mathrm{T}}\left(\mathbf{N}_{x}^{-1}\right)^{\mathrm{T}} \mathbf{N}_{x}^{-1} \mathbf{x}=\rho^{2}$, from which we derive the analytical expression (2.13).

The eigenvalues $d_{1}$ and $d_{2}$ of the symmetric matrix $\mathbf{N}:=\left(\mathbf{N}_{x}^{-1}\right)^{\mathrm{T}} \mathbf{N}_{x}^{-1} \in \mathbb{R}_{\mathrm{sym}}^{2 \times 2}$ are calculated (after some algebra) as

$$
d_{i}=\frac{\csc ^{2} \omega}{2 u^{2}}\left[u^{2}+1+(-1)^{i} \sqrt{u^{4}+1+2 u^{2} \cos 2 \omega}\right], \quad i=1,2,
$$

with $0<\left(u^{2}-1\right)^{2}=u^{4}-2 u^{2}+1<u^{4}+1+2 u^{2} \cos 2 \omega$ for every $u>0$, and therefore the requested major and minor radius of the ellipse $S_{x}(C)$ are correspond to the eigenvalues $d_{2}$ and $d_{1}$ respectively (as $\left.d_{2}<d_{1}\right)$, i.e. $a=\rho / \sqrt{d_{2}}$ and $b=\rho / \sqrt{d_{1}}$, which are given by (2.14).

Moreover, the non-unitary orthogonal eigenvectors $\mathbf{v}_{i}=\left(v_{i ; 1}, v_{i ; 2}\right)^{\mathrm{T}} \in \mathbb{R}^{2 \times 1}$ that correspond to its eigenvalues $d_{i}, i=1,2$ are then calculated through (2.16) and (after some algebra), are found to be

$$
\mathbf{v}_{i}=\left(-\frac{1+2 u^{2} \cos ^{2} \omega+(-1)^{i-1} \sqrt{u^{4}+1+2 u^{2} \cos 2 \omega}}{u^{2} \sin 2 \omega}, 1\right), \quad i=1,2 .
$$

Hence, as the major radius $a$ corresponds to the eigenvalue $d_{2}$, the directive angle $\theta$ of the ellipse $S_{x}(C)$ is then of the form $\theta=\angle\left(\mathbf{e}_{1}, \mathbf{v}_{2}\right)=\arctan \left(v_{2 ; 2} / v_{2 ; 1}\right)$ and it is given by $(2.15)$.

Notice that the assumption $\tan \theta>\tan \omega>0$ yields, through (2.15), that $u^{2}-1>\sqrt{u^{4}+1+2 u^{2} \cos 2 \omega}$ which cannot hold (as it then yields that $\cos ^{2} \omega<0$ ). Therefore, $\tan \theta<\tan \omega$ for $\omega>0$, and respectively, $\tan \theta>\tan \omega$ for $\omega<0$, i.e. $|\theta|<|\omega|$.

The following example clarifies the $x$ - and $y$-shear transformations of a circle, which provides two ellipses having a common radius. It is also the basis for some other examples provided hereafter.

Example 2.1. Let $C$ be a circle of radius $\rho:=10$ centered at the origin $O$ of an o.c.s. spanned by the orthonormal vector base $\mathscr{B}=\left\{\mathbf{e}_{1}, \mathbf{e}_{2}\right\}$, and its points $P, Q \in C$, with $P(\rho, 0), Q(0, \rho)$, which define $C^{\prime}$ s orthogonal radii $O P$ and $O Q$. These radii can be considering as $C^{\prime}$ 's trivially conjugate radii, with $O P \perp O Q$ and $|O P|=|O Q|=\rho=10$. Let also a third point $R$ with $|O R|=r:=15$ and angle $\measuredangle\left(\mathbf{e}_{1}, O R\right)=\omega:=\pi / 6\left(=30^{\circ}\right)$.

The $y$-shear transformation $S_{y}$ which transforms point $P(\rho, 0) \in C$ into $R$ while preserves point $Q \in C$, is the one having shearing vector $\mathbf{u}=\rho^{-1} O R$ (of length $u=|\mathbf{u}|=r / \rho=3 / 2$ and angle $\omega=\pi / 6$ ). Indeed, it can be shown through (2.4) that $S_{y}(Q)=Q$ and $S_{y}(P)=R$, with $u$ and $\omega$ values as above. Therefore, the $y$-sheared circle corresponds to a concentric ellipse, say $E_{y}:=S_{y}(C)$, for which $O R$ and $O Q$ are its two conjugate radii. The conjugality of radii $O P$ and $O Q$ is derived from the fact that the $y$-shear (as well as $x$-shears) is, in principle, an affine transformation and, as such, it preserves the parallelism on the plane; see Fig. 4 where the circumscribing square frame of the circle $C$ is transformed through $S_{y}$ into a parallelogram circumscribing $E_{y}=S_{y}(C)$.

Working similarly, the $x$-shear transformation $S_{x}$ which transforms $Q(\rho, 0) \in C$ into $R$ while preserves point $P \in C$, is the one having the same shearing vector $\mathbf{u}$ as $S_{y}$. From (2.3), it holds that $S_{x}(Q)=R$ and $S_{x}(P)=R$. Thus, the $x$-sheared circle corresponds to a concentric ellipse, say $E_{x}:=S_{x}(C)$, for which $O R$ and $O P$ are its two conjugate radii; see also Fig. 4.

Therefore, the given bundle of the three line segments $O P, O Q$ and $O R$ corresponds to a bundle of three common (conjugate per pair) radii which define the ellipses $E_{x}, E y$ and, trivially, the circle $C$. In particular, we may write: $E_{x}=E_{x}(O P, O R), E_{y}=E_{y}(O Q, O R)$ and $C=C(O P, O Q)$, meaning that $E_{x}$ is defined by (its conjugate radii) $O P$ and $O Q, E_{y}$ is defined by $Q P$ and $O R$, while $C$ is trivially defined by $O P$ and $O Q$. Figure 4 provides again a clarification of the above discussion.

For the specific example, the analytical expression of ellipse $E_{y}$ is of the form $E_{y}: 25 x^{2}-18 \sqrt{3} x y+27 y^{2}=2700$, due to (2.5) where it was set $u:=3 / 2$, while its principal radii $0<b_{y}<a_{y}$ are given by $a_{y}=\frac{5}{2} \sqrt{26+2 \sqrt{61}} \approx$ 16.1285 and $b_{y}=\frac{5}{2} \sqrt{26-2 \sqrt{61}} \approx 8.0543$, through (2.6). From (2.7), $E_{y}$ 's directive angle $\theta_{y}$ is then given by $\theta_{y}=\arctan \left\{\frac{\sqrt{3}}{27}(2 \sqrt{61}-1)\right\} \approx 43.1648^{\circ}$.

The analytical expression of the ellipse $E_{x}$ is of the form $E_{x}: 9 x^{2}-18 \sqrt{3} x y+43 y^{2}=900$, due to (2.13) where was also set $u:=3 / 2$, while its principal radii $0<b_{x}<a_{x}$ are given by $a_{x}=\frac{5}{2}(\sqrt{19}+\sqrt{7}) \approx 17.5116$ and $b_{x}=\frac{5}{2}(\sqrt{19}-\sqrt{7}) \approx 4.2829$, through (2.14). From (2.15), the $E_{x}$ 's directive angle $\theta_{x}$ is given by $\theta_{x}=$ $\arctan \left\{\frac{\sqrt{3}}{27}(2 \sqrt{133}-17)\right\} \approx 21.2599^{\circ}$.

Figure 4 visualizes exactly Example 2.1 by depicting the circle $C$ together with its $x$ - and $y$-shears, i.e. the ellipses $E_{x}=S_{x}(C)$ and $E_{y}=S_{y}(C)$. The $S_{x}$ and $S_{y}$ axis shears (of shearing vector $\mathbf{u}=\rho^{-1} O R$ ) are also illustrated 
by presenting the square frame around $C$ and its transformed parallelograms, through $S_{x}$ and $S_{y}$, around the ellipses $E_{x}$ and $E_{y}$ respectively. The corresponding foci $F_{x}$ and $F_{y}$ are also depicted.

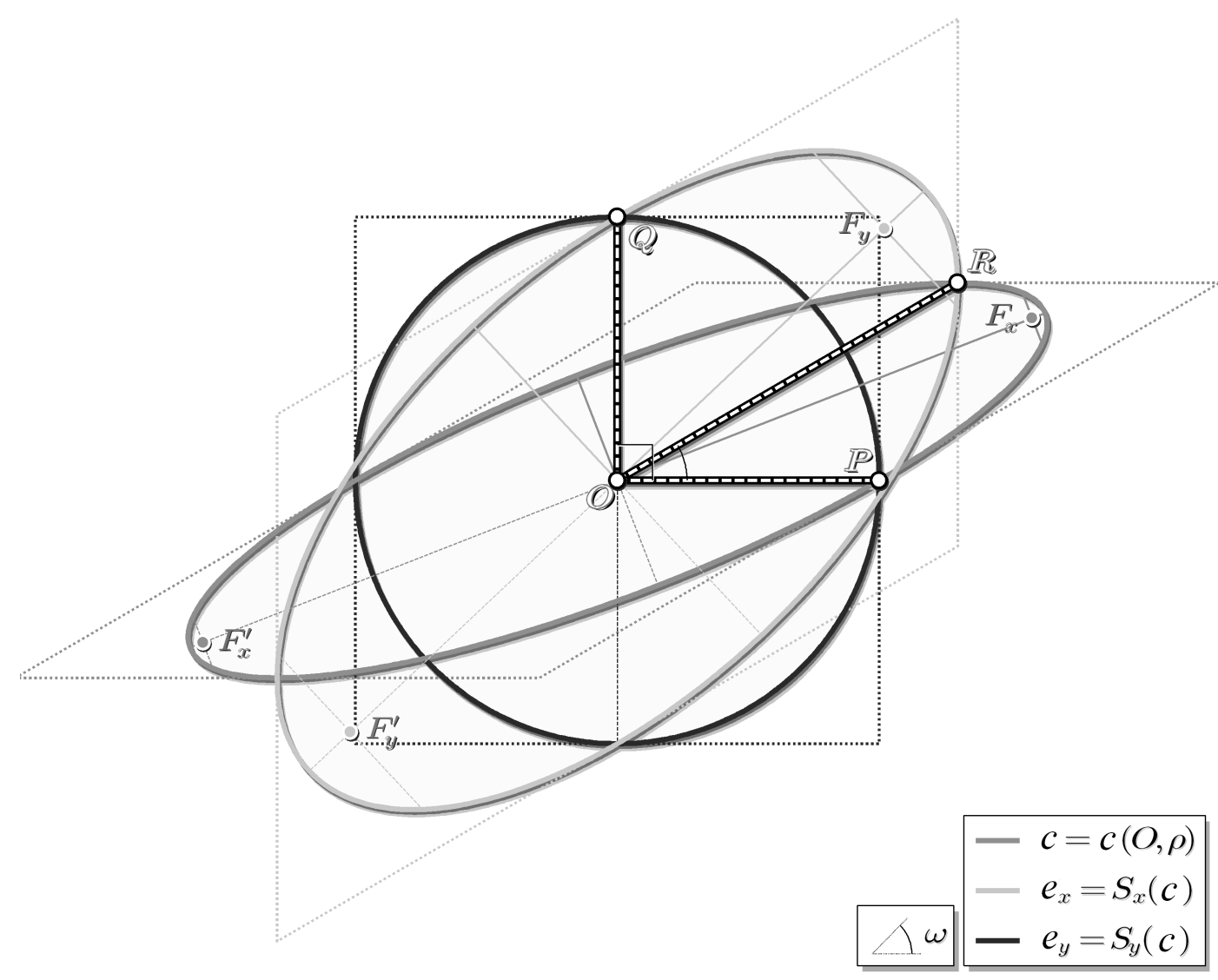

Figure 4. Circle $C$ and its $x$-and $y$-shears $E_{x}$ and $E_{y}$.

\section{Common Tangential Ellipses}

In this section we deliver the main results addressing the problem of finding a common tangential ellipse around three given"mutually conjugate" ellipses which are described as follows:

Definition 3.1. Three concentric and coplanar ellipses shall called mutually conjugate (with each other) when each of them is defined by a pair of two conjugate radii taken from a bundle of three given (non-degenerated) line segments, where only two of these segments are permitted to coincide. These line segments as above shall called as the three mutually conjugate radii corresponding to the three mutually conjugate ellipses.

Recall Example 2.1 where $E_{x}, E_{y}$ and $C$ (all centered at $O$ ) are indeed three mutually conjugate ellipses defined by their three given mutually conjugate radii $O P, O Q$ and $O R$, such that $E_{x}:=E_{x}(O P, O R), E_{y}:=$ $E_{y}(O Q, O R)$ and $C:=C(O P, O Q)$. Furthermore, we shall extend our investigation, in the sense that we shall derive (not one but) all the common tangential ellipses (of three given mutually conjugate ellipses) that can exist. This Section is divided into two sub-Sections regarding the following cases:

- The orthogonal case, in which the existence of the common tangential ellipses of a given circle and two ellipses, mutually conjugate with each other, is investigated, and

- The general case, based on the orthogonal one, where the existence of the common tangential ellipses around three given non-circular mutually conjugate ellipses is examined.

Before these subs-Sections we state and prove the following Lemma which is needed for our study. This Lemma investigates the form of a "tangential ellipse" of a given ellipse $E$, i.e. a concentric (to $E$ ) ellipse, say 
$\bar{E}$, which is tangent to $E$. The ellipses $\bar{E}$ and $E$ are then intersect with each other at two (in total) distinct diametrical (contact) points, on which their corresponding two tangent lines coincide.

Lemma 3.1. Consider a tangential ellipse $\bar{E}$ of a given ellipse $E$ and $\theta \in[-\pi, \pi]$ be a given angle between the major axes of $E$ and $\bar{E}$. When $\bar{E}$ 's minor radius $\bar{b}$ is given, then its corresponding major radius $\bar{a}$ satisfies the relation

$$
A \bar{a}^{4}+B \bar{a}^{2}+C=0
$$

where

$$
\begin{aligned}
& A:=4 \bar{b}^{2} \lambda^{-2}\left(\bar{b}^{2}-a^{2}\right) \cos ^{2} \theta+4 a^{2} \bar{b}^{2}\left(\lambda^{-2}-1\right) \cos ^{4} \theta, \\
& B:=a^{2} \bar{b}^{4}\left(1-\lambda^{-2}\right) \cos ^{2} 2 \theta+2 \bar{b}^{2}\left(a^{4}-\lambda^{-2} \bar{b}^{4}\right) \cos 2 \theta+2 \bar{b}^{2}\left[a^{4}+\lambda^{-2} \bar{b}^{4}-a^{2} \bar{b}^{2}\left(1+\lambda^{-2}\right)\right], \\
& C:=a^{2} \bar{b}^{6}\left(1-\lambda^{-2}\right) \sin ^{2} 2 \theta+4 a^{2} \bar{b}^{4}\left(a^{2}-\bar{b}^{2}\right) \sin ^{2} \theta,
\end{aligned}
$$

with $0<b<a$ being the $E$ 's given principal radii and $\lambda$ being its corresponding aspect ratio, i.e. $\lambda:=b / a$. The analytical expression of the requested $\bar{E}$, on an o.c.s. spanned by the principal axes of the given ellipse $E$, is then formulated by

$$
\bar{E}:\left(\bar{a}^{2} \sin ^{2} \theta+\bar{b}^{2} \cos ^{2} \theta\right) x^{2}-\left(\bar{a}^{2}-\bar{b}^{2}\right)(\sin 2 \theta) x y+\left(\bar{a}^{2} \cos ^{2} \theta+\bar{b}^{2} \sin ^{2} \theta\right) y^{2}=\bar{a}^{2} .
$$

Proof. We consider the ellipse $E$ centered at the origin $O$ of an o.c.s. which is spanned by its principal axes, i.e. the $E^{\prime}$ s major and minor axis are form, respectively, the o.c.s.'s horizontal and vertical axis. Hence, the ellipse $E$ (in this o.c.s.), adopts its canonical form

$$
E:(x / a)^{2}+(y / b)^{2}=1
$$

The tangential ellipse $\bar{E}$ of $E$, which (its major axis) forms a given angle $\theta$ with (the major axis of) $E$, adopts two diametrical points $P$ and $P^{\prime}$ in common with $E$. Therefore, $\bar{E}$ can be expressed in the form of the ellipse (centered at the origin $O$ ) as in (2.1), and thus (3.3) holds, with $0<\bar{b}<\bar{a}$ being the $\bar{E}^{\prime}$ s principal radii. Notice that $\theta$ is indeed the directive angle of $\bar{E}$ (with respect to the adopted o.c.s. as above) as it coincides with the given angle $\theta$ between the major axes of $E$ and $\bar{E}$ ).

Assumed now that the $\bar{E}^{\prime}$ 's minor radius $\bar{b}$ is given, we shall provide in the following the $\bar{E}^{\prime}$ s major axis $\bar{a}$, and thus the tangential ellipse $\bar{E}$ of a given ellipse $E$ (in the direction of the given angle $\theta$ ) can then be calculated easily though (3.3). Let $\lambda:=b / a$ and $\bar{\lambda}:=\bar{b} / \bar{a}$ be the aspect ratios of the ellipses $E$ and $\bar{E}$ respectively. We consider the contact point $P\left(x_{0}, y_{0}\right) \in E \cap \bar{E}$ which lies on the o.c.s.'s upper semi-plane, i.e. $y_{0} \geq 0$. Solving (3.4) and (3.3), with respect to $y$, we obtain

$$
\begin{aligned}
& y_{0}=y_{E}\left(x_{0}\right):=\lambda \sqrt{a^{2}-x_{0}^{2}}, \quad x_{0} \in[-a, a], \text { and } \\
& y_{0}=y_{\bar{E}}\left(x_{0}\right):=\frac{\frac{1}{2}\left(\bar{\lambda}^{-2}-1\right) x_{0} \sin 2 \theta+\bar{\lambda}^{-1} \sqrt{\bar{\lambda}^{-2} \bar{b}^{2} \cos ^{2} \theta+\bar{b}^{2} \sin ^{2} \theta-x_{0}^{2}}}{\bar{\lambda}^{-2} \cos ^{2} \theta+\sin ^{2} \theta},
\end{aligned}
$$

where, for the latter function $y_{\bar{E}}\left(x_{0}\right)$, it is assumed that $x_{0} \in[-t, t]$ with the value $t:=\bar{b}^{2}\left(\bar{\lambda}^{-2} \cos ^{2} \theta+\sin ^{2} \theta\right)$. Equating the right-hand side of (3.5a) and (3.5b), it holds that

$$
\lambda K \sqrt{a^{2}-x_{0}^{2}}=L x_{0}+\bar{\lambda}^{-1} \bar{b}^{2} \sqrt{K-x_{0}^{2}}
$$

where

$$
\begin{aligned}
K & :=\bar{\lambda}^{-2} \bar{b}^{2} \cos ^{2} \theta+\bar{b}^{2} \sin ^{2} \theta=\bar{b}^{2}\left[1+\left(\bar{\lambda}^{-2}-1\right) \cos ^{2} \theta\right]>0 \text { and } \\
L & :=\frac{1}{2} \bar{b}^{2}\left(\bar{\lambda}^{-2}-1\right) \sin 2 \theta=\left(K-\bar{b}^{2}\right) \tan \theta .
\end{aligned}
$$

In order the curves $y=y_{E}(x)$ and $y=y_{\bar{E}}(x)$, as defined in (3.5a) and (3.5b), to be tangent to each other at their common point $P\left(x_{0}, y_{0}\right)$ (and hence to have a common tangent line on $P$ ), the derivatives of $y_{E}=y_{E}(x)$ and $y_{\bar{E}}=y_{\bar{E}}(x)$ must coincide at $x=x_{0}$ with $x_{0}$ satisfying (3.6). The derivative of (3.6), with respect to $x=x_{0}$, yields

$$
\lambda K \frac{x_{0}}{\sqrt{a^{2}-x_{0}^{2}}}=-L+\bar{b}^{2} \frac{x_{0}}{\bar{\lambda} \sqrt{K-x_{0}^{2}}} .
$$


Solving (3.6) and (3.8) in terms of $\sqrt{K-x_{0}^{2}}$, we get

$$
\frac{\lambda \bar{\lambda} K}{\bar{b}^{2}} \sqrt{a^{2}-x_{0}^{2}}-\frac{L \bar{\lambda} x_{0}}{\bar{b}^{2}}=\sqrt{K-x_{0}^{2}}=\frac{\bar{\lambda}^{-1} \bar{b}^{2} x_{0} \sqrt{a^{2}-x_{0}^{2}}}{\lambda K x_{0}+L \sqrt{a^{2}-x_{0}^{2}}} .
$$

By multiplication of the left and right side of (3.9), we obtain the squared middle expression of (3.9) of the form

$$
\begin{gathered}
K-x_{0}^{2}=\frac{\left(\lambda K \sqrt{a^{2}-x_{0}^{2}}-L x_{0}\right) x_{0} \sqrt{a^{2}-x_{0}^{2}}}{\lambda K x_{0}+L \sqrt{a^{2}-x_{0}^{2}}} \text { or } \\
\sqrt{a^{2}-x_{0}^{2}}=\lambda \frac{a^{2}-K}{L} x_{0} .
\end{gathered}
$$

Moreover, relation (3.8) can be written as

$$
\lambda K x_{0}+L \sqrt{a^{2}-x_{0}^{2}}=\bar{\lambda}^{-1} \bar{b}^{2} x_{0} \sqrt{\frac{a^{2}-x_{0}^{2}}{K-x_{0}^{2}}},
$$

and applying (3.8) to the left hand side of the above we get

$$
\lambda \bar{\lambda} a^{2} \sqrt{K-x_{0}^{2}}=\bar{b}^{2} \sqrt{a^{2}-x_{0}^{2}},
$$

while substituting (3.6) again to the right-hand side of (3.11), we obtain

$$
\sqrt{K-x_{0}^{2}}=\frac{\bar{b}^{2}}{L \bar{\lambda} a^{2}}\left(a^{2}-K\right) x_{0} .
$$

Applying (3.10) and (3.12) into (3.6) we have $\left[K b^{2}-\left(\bar{\lambda}^{-1} \bar{b}^{2}\right)^{2}\right]\left(a^{2}-K\right)=L^{2} a^{2}$, and using (3.7b),

$$
\lambda^{2} a^{2} K-\lambda^{2} K^{2}-\left(\bar{\lambda}^{-1} \bar{b}^{2}\right)^{2}+\left(\bar{\lambda}^{-1} \bar{b}^{2} a^{-1}\right)^{2} K=\left(K^{2}-2 K \bar{b}^{2}+\bar{b}^{4}\right) \tan ^{2} \theta,
$$

i.e.

$$
K^{2} b^{2}\left(1+\lambda^{-2} \tan ^{2} \theta\right)-K\left[\left(\bar{\lambda}^{-1} \bar{b}^{2}\right)^{2}+a^{2} b^{2}+2 a^{2} \bar{b}^{2} \tan ^{2} \theta\right]+a^{2} \bar{b}^{4}\left(\bar{\lambda}^{-2}+\tan ^{2} \theta\right)=0 .
$$

Finally, by substitution of (3.7a) into the above (3.14) we obtain the biquadratic polynomial relation (3.1) with respect of $\bar{E}$ 's requested major radius $\bar{a}$.

\subsection{The orthogonal case}

We consider the following lemma which investigates the existence of a c.t.e. of a circle and its two $x$ - and $y$-shear transformations.

Lemma 3.2. Let $C$ be a circle of radius $\rho>0$ centered at the origin $O$ of an o.c.s. The circle $C$ as well as the ellipses $E_{x}$ and $E_{y}$, produced by $x$-and $y$-shears of $C$ with the same given shearing vector $\mathbf{u}$ of length $u>0$ and angle $\omega \in[-\pi, \pi]$, can always adopt $a$ c.t.e. $E$ in the direction of the vector $\mathbf{u}$ (i.e. $E^{\prime}$ 's directive angle is $\omega$ ). The principal radii $0<b<a$ of the requested c.t.e. $E$ are then given by $a=\rho \sqrt{u^{2}+1}$ and $b=\rho$, with $E^{\prime}$ s foci semi-distance (or linear eccentricity) is being $f=u \rho$, as $E$ is analytically expressed (in the adopted o.c.s.) by

$$
E:\left[\left(u^{2}+1\right) \sin ^{2} \omega+\cos ^{2} \omega\right] x^{2}-u^{2}(\sin 2 \omega) x y+\left[\left(u^{2}+1\right) \cos ^{2} \omega+\sin ^{2} \omega\right] y^{2}=\rho^{2}\left(u^{2}+1\right) .
$$

Proof. Consider an orthonormal vector base $\mathscr{B}$ of an o.c.s. of origin $O$ and let $S_{x}$ and $S_{y}$ be the $x$ - and $y$-shear transformations with the same shearing vector (of length $u>0$ and angle $\omega$ ). Then, according to Lemmas 2.3 and 2.2, the $x$ - and $y$-sheared circles $E_{x}:=S_{x}(C)$ and $E_{y}:=S_{y}(C)$ are ellipses centered also at $O$ with their corresponding principal radii $0<b_{x}<a_{x}$ and $0<b_{y}<a_{y}$ given by (2.14) and (2.6). The directive angles $\theta_{x}, \theta_{y} \in[-\pi, \pi]$ of the tangential ellipses $E_{x}$ and $E_{y}$ (with respect to the o.c.s.'s coordinate axes) are then given by (2.15) and (2.7) respectively. Let also $\bar{E}_{x}$ and $\bar{E}_{y}$ be the (concentric) tangential ellipses of $E_{x}$ and $E_{y}$ respectively, 
adopted in the same direction as the common shearing vector (i.e. their directive angles are both $\omega$ ), and having principal radii $0<\bar{b}_{x}<\bar{a}_{x}$ and $0<\bar{b}_{y}<\bar{a}_{y}$ respectively.

We are shall now investigate the form of tangential ellipses $\bar{E}_{x}$ and $\bar{E}_{y}$ of $E_{x}$ and $E_{y}$ in the direction of $\omega$, such that both $\bar{E}_{x}$ and $\bar{E}_{y}$ are also tangent to the circle $C$. Thus, their minor radii $\bar{b}_{x}=\bar{b}_{y}=\rho$, while their directive angles $\bar{\theta}_{x}=\bar{\theta}_{y}=\omega$. In order to apply Lemma 3.1, we consider as angle $\theta$ (which is the angle between the major axes of $E_{x}$ and $\bar{E}_{x}$ ) the difference $\theta:=\bar{\theta}_{x}-\theta_{x}=\omega-\theta_{x}$, while for the angle between (the major axes of) $E_{y}$ and $\bar{E}_{y}$ we must consider, respectively, $\theta:=\bar{\theta}_{y}-\theta_{y}=\omega-\theta_{y}$.

Let $k:=\bar{b}_{x} / b_{x}$, while $\lambda_{x}:=b_{x} / a_{x}$ and $\bar{\lambda}_{x}:=\bar{b}_{x} / \bar{a}_{x}$ denote the aspect ratios of $E_{x}$ and $\bar{E}_{x}$ respectively. Relation (3.1), through (3.7a), yields

$k^{2}\left(1+\lambda_{x}^{-2} \tan ^{2} \theta\right)\left[1+\left(\bar{\lambda}_{x}^{-2}-1\right) \cos ^{2} \theta\right]^{2}+\lambda_{x}^{-2} k^{2}\left(\bar{\lambda}_{x}^{-2}+\tan ^{2} \theta\right)-\left[\bar{\lambda}_{x}^{-2} k^{4}+\lambda_{x}^{-2}\left(1+2 k^{2} \tan ^{2} \theta\right)\right]\left[1+\left(\bar{\lambda}_{x}^{-2}-1\right) \cos ^{2} \theta\right]=0$, or equivalently,

$$
\lambda_{x}^{-2}\left(\bar{\lambda}_{x}^{-2} \cos ^{2} \theta+\sin ^{2} \theta\right)\left\{\bar{\lambda}_{x}^{-2} k^{4}-\left[\left(\bar{\lambda}_{x}^{-2}+\lambda_{x}^{-2}\right) \cos ^{2} \theta+\left(1+\bar{\lambda}_{x}^{-2} \lambda_{x}^{-2}\right) \sin ^{2} \theta\right] k^{2}+\lambda_{x}^{-2}\right\}=0 .
$$

and hence we derive the following biquadratic polynomial with respect of $k$,

$$
\bar{\lambda}_{x}^{-2} k^{4}-\left[\left(\bar{\lambda}_{x}^{-2}+\lambda_{x}^{-2}\right) \cos ^{2} \theta+\left(1+\bar{\lambda}_{x}^{-2} \lambda_{x}^{-2}\right) \sin ^{2} \theta\right] k^{2}+\lambda_{x}^{-2}=0 .
$$

Substituting $k=\bar{b}_{x} / b_{x}=\rho / b_{x}$ (as $\bar{b}_{x}=\rho$ were assumed), the above polynomial (3.16) yields

$$
\bar{\lambda}_{x}^{-2} \rho^{4}-\left[1+\bar{\lambda}_{x}^{-2} \lambda_{x}^{-2}-\left(\bar{\lambda}_{x}^{-2}-1\right)\left(\lambda_{x}^{-2}-1\right) \cos ^{2} \theta\right] \rho^{2} b_{x}^{2}+\lambda_{x}^{-2} b_{x}^{4}=0,
$$

and solving (3.16) with respect to $\bar{\lambda}_{x}^{-2}$ we get

$$
\bar{\lambda}_{x}^{-2}=b_{x}^{2} \rho^{-2} \frac{b_{x}^{2} \lambda_{x}^{-2}-\rho^{2}-\rho^{2}\left(\lambda_{x}^{-2}-1\right) \cos ^{2} \theta}{b_{x}^{2} \lambda_{x}^{-2}-\rho^{2}-b_{x}^{2}\left(\lambda_{x}^{-2}-1\right) \cos ^{2} \theta} .
$$

From (2.14) we have

$$
b_{x}^{2} \lambda_{x}^{-2}=\frac{8 u^{4} \rho^{2} \sin ^{4} \omega}{\delta_{x}^{2}\left(u^{2}+1+\sqrt{u^{4}+1+2 u^{2} \cos 2 \omega}\right)}=2 \delta_{x}^{-1} u^{2} \rho^{2} \sin ^{2} \omega
$$

where

$$
\delta_{x}:=u^{2}+1-\sqrt{u^{4}+1+2 u^{2} \cos 2 \omega} .
$$

and thus, by substitution of (3.18) into (3.17) and then applying $b_{x}$ as in (2.14), we obtain

$$
\bar{\lambda}_{x}^{2}=\frac{\rho^{2} \delta_{x}\left[u^{2}-1+\left(u^{2}+1-\delta_{x}\right)\left(1-2 \cos ^{2} \theta\right)\right]}{2 \rho^{2} u^{2} \delta_{x} \sin ^{2} \omega-\rho^{2} \delta_{x}^{2} \sin ^{2} \theta-4 \rho^{2} u^{2} \sin ^{2} \omega \cos ^{2} \theta .}
$$

Moreover, substituting $\theta_{x}$ from (2.15) to the relation

$$
\cos ^{2} \theta=\frac{1}{1+\tan ^{2}\left(\omega-\theta_{x}\right)}=\frac{\left(1+\tan \omega \tan \theta_{x}\right)^{2}}{\left(1+\tan \omega \tan \theta_{x}\right)^{2}+\left(\tan \omega-\tan \theta_{x}\right)^{2}},
$$

as $\theta:=\omega-\theta_{x}$, we obtain that through (3.19),

$$
\cos ^{2} \theta=\frac{\left(2 u^{2}+2-\delta_{x}\right)^{2}}{\left(2 u^{2}+2-\delta_{x}\right)^{2}+\left(\tan ^{2} \omega\right)\left(2-\delta_{x}\right)^{2}} .
$$

By substitution of the above $\cos ^{2} \theta$ into (3.20) we obtain (after some algebra) that

$$
\bar{\lambda}_{x}^{2}=\frac{\delta_{x}\left(u^{2}-1-\delta_{x}\right)\left[2\left(u^{2}+1\right)-\delta_{x}\right]^{2}\left(1-\sin ^{2} \omega\right)+\delta_{x}\left(u^{2}-1+\delta_{x}\right)\left(\delta_{x}-2\right)^{2} \sin ^{2} \omega}{\left[\delta_{x}\left(\delta_{x}-2\right)\left(2 u^{2} \sin ^{2} \omega-\delta_{x}\right)+2 u^{2}\left[2\left(u^{2}+1\right)-\delta_{x}\right]^{2}\left(1-\sin ^{2} \omega\right)\right]\left(\delta_{x}-2\right) \sin ^{2} \omega}
$$


From (3.19) we have

$$
\sin ^{2} \omega=1-\cos ^{2} \omega=1-\frac{1}{2}(1+\cos 2 \omega)=1+\frac{1}{4 u^{2}}\left(\delta_{x}-2\right)\left(2 u^{2}-\delta_{x}\right),
$$

as it is easy to see, through (3.19), that $0<\delta_{x}<2$ and $\delta_{x}<2 u^{2}$. Substituting (3.23) into (3.22), we finally derive (after a series of simplifications) that $\bar{\lambda}_{x}^{2}=u^{2}+1$, and hence the major radius of $\bar{E}_{x}$ is given by $\bar{a}_{x}=\rho \sqrt{u^{2}+1}$, as $\bar{b}_{x}=\rho$ was assumed.

Working similarly for the case of the tangential ellipse $\bar{E}_{y}$ we obtain the its aspect ratio

$$
\begin{gathered}
\bar{\lambda}_{y}^{-2}=b_{y}^{2} \rho^{-2} \frac{b_{y}^{2} \lambda_{y}^{-2}-\rho^{2}-\rho^{2}\left(\lambda_{y}^{-2}-1\right) \cos ^{2} \theta}{b_{y}^{2} \lambda_{y}^{-2} \sin ^{2} \theta-\rho^{2}+b_{y}^{2} \cos ^{2} \theta} \text { or } \\
\bar{\lambda}_{y}^{2}=\frac{\rho^{2} \delta_{y}\left[u^{2}-1+\left(u^{2}+1-\delta_{y}\right)\left(1-2 \cos ^{2} \theta\right)\right]}{2 \rho^{2} u^{2} \delta_{y} \cos ^{2} \omega-\rho^{2} \delta_{y}^{2} \sin ^{2} \theta-4 \rho^{2} u^{2} \cos ^{2} \omega \cos ^{2} \theta}, \text { where } \\
\delta_{y}:=u^{2}+1-\sqrt{u^{4}+1-2 u^{2} \cos 2 \omega} .
\end{gathered}
$$

Then, after some algebra, it holds also that $\bar{\lambda}_{y}^{2}=u^{2}+1$, and hence the major radius of $\bar{E}_{y}$ is given by $\bar{a}_{y}=\rho \sqrt{u^{2}+1}$, as $\bar{b}_{y}=\rho$ was also assumed.

Therefore, the principal radii of $\bar{E}_{x}$ and $\bar{E}_{y}$ coincide, as $\bar{a}_{x}=\bar{a}_{y}=\rho \sqrt{u^{2}+1}$ and $\bar{b}_{x}=\bar{b}_{y}=\rho$, and hence the ellipses $\bar{E}_{x}$ and $\bar{E}_{y}$ are of the same shape. Moreover, as their directive angles $\bar{\theta}_{x}$ and $\bar{\theta}_{y}$ are both assumed to be $\omega$, it is clear that $\bar{E}_{x}=\bar{E}_{y}$. We can then denote with $E:=\bar{E}_{x}=\bar{E}_{y}$ the c.t.e. of $E_{x}, E_{y}$ and $C$. The directive angle $\theta$ of $E$ is $\omega$ and its principal radii $0<b<a$ are of the form $a=\rho \sqrt{u^{2}+1}$ and $b=\rho$. The foci semi-distance of $E$ is formulated by $f:=\sqrt{a^{2}-b^{2}}=u \rho$. The analytical expression of the c.t.e. $E$ is finally given by setting $\theta:=\omega$ into (2.1), and thus (3.15) is finally derived.

The following Theorem proves the existence of a c.t.e. of a circle and two ellipses mutually conjugate with each other.

Theorem 3.1. Consider a circle $C$ of radius $\rho>0$ centered at point $O$, and two ellipses $E_{1}$ and $E_{2}$ such that $E_{1}, E_{2}$ and $C$ correspond to three given mutually conjugate ellipses. Hence, these ellipses are defined by a bundle of three given mutually conjugate radii, say $O P, O Q$ and $O R$, such that $C=C(O P, O Q), E_{1}=E_{1}(O P, O R)$ and $E_{2}=E_{2}(O Q, O R)$, with $|O P|=|O Q|=\rho$ and $O P \perp O Q$, as $C$ is a circle. These radii are fully determined by the given length $r=|O R|$ and angle $\omega=\measuredangle(O P, O R) \in[-\pi, \pi]$. Then, a common tangential ellipse $E$, of $E_{1}, E_{2}$ and $C$, always exists in the direction of the non-orthogonal radius $O R$ (i.e. the major radius of $E$ is spanned by $O R$ ), while point $R$ is one of the $E^{\prime}$ s foci. The principal radii $0<b<a$ of $E$ are then given by $a=\sqrt{\rho^{2}+r^{2}}$ and $b=\rho$, while its eccentricity and foci semi-distance are $\varepsilon=r / \sqrt{\rho^{2}+r^{2}}$ and $f=r$ respectively. This c.t.e. $E$ is analytically expressed by

$$
E:\left[\left(\rho^{2}+r^{2}\right) \sin ^{2} \omega+\rho^{2} \cos ^{2} \omega\right] x^{2}-r^{2}(\sin 2 \omega) x y+\left[\left(\rho^{2}+r^{2}\right) \cos ^{2} \omega+\rho^{2} \sin ^{2} \omega\right] y^{2}=\rho^{2}\left(\rho^{2}+r^{2}\right),
$$

in an o.c.s. spanned by the orthogonal radii $O P$ and $O Q$.

The two diametrical (common) contact points $T_{1}\left(x_{1}, y_{1}\right), x_{1}>0$, and $T_{1}^{\prime}\left(-x_{1},-y_{1}\right)$ between $E_{1}$ and its tangential ellipse $E$, are then given by

$$
x_{1}=\sqrt{\rho^{2}+r^{2} \cos ^{2} \omega} \text { and } y_{1}=\frac{r^{2} \sin 2 \omega}{2 \sqrt{\rho^{2}+r^{2} \cos ^{2} \omega}},
$$

while their corresponding two common tangent lines $t_{1}$ and $t_{1}^{\prime}$ (at points $T_{1}$ and $T_{1}^{\prime}$ respectively) are being parallel to $O Q$ (which spans the o.c.s.'s vertical axis $y^{\prime} O y$ ), i.e. $t_{1}: x=x_{1}$ and $t_{1}^{\prime}: x=-x_{1}$.

For the two diametrical contact points $T_{2}\left(x_{2}, y_{2}\right), y_{2}>0$, and $T_{1}^{\prime}\left(-x_{2},-y_{2}\right)$ between $E_{2}$ and its tangential ellipse $E$, we have

$$
x_{2}=\frac{r^{2} \sin 2 \omega}{2 \sqrt{\rho^{2}+r^{2} \sin ^{2} \omega}} \text { and } y_{2}=\sqrt{\rho^{2}+r^{2} \sin ^{2} \omega},
$$

while their corresponding two common tangent lines $t_{2}$ and $t_{2}^{\prime}$ (at points $T_{2}$ and $T_{2}^{\prime}$ respectively) are being parallel to $O P$ (which spans the o.c.s.'s horizontal axis $x^{\prime} O x$ ), i.e. $t_{2}: y=y_{2}$ and $t_{2}^{\prime}: y=-y_{2}$. 
Finally, for the last two contact points $T_{3}\left(x_{3}, y_{3}\right)$ and $T_{3}^{\prime}\left(-x_{3},-y_{3}\right)$ between circle $C$ and its tangential ellipse $E$, it holds that

$$
x_{3}=-\rho \sin \omega \text { and } y_{3}=\rho \cos \omega,
$$

while their corresponding two tangent lines $t_{3}$ and $t_{3}^{\prime}$ (at points $T_{3}$ and $T_{3}^{\prime}$ respectively) are being parallel to the nonorthogonal radii $O R$, as they are given by

$$
t_{3}: y \cos \omega-x \sin \omega=\rho \text { and } t_{3}^{\prime}: x \sin \omega-y \cos \omega=\rho .
$$

Proof. Consider an o.c.s. with origin $O$ where its horizontal and vertical axes are spanned by the given orthogonal vectors $O P$ and $O Q$. Let $\mathscr{B}=\left\{\mathbf{e}_{1}, \mathbf{e}_{2}\right\}$ be the corresponding orthonormal vector base. Hence $P_{\mathscr{B}}(\rho, 0)$ and $Q_{\mathscr{B}}(0, \rho)$. Let $S_{x}$ be the $x$-shear transformation with shearing vector $\mathbf{u}:=\rho^{-1} O R$, which transforms point $Q \in C$ into $R$. Indeed, from (2.3) and setting $u:=|\mathbf{u}|=r / \rho$, we obtain $S_{x}(Q)=R$. Notice also that $S_{x}(P)=P$ (as the horizontal axis $x^{\prime} O x$ is invariant under $S_{x}$ ), and hence $P \in C \cap E_{1}$. Therefore, $S_{x}(C)=E_{1}$ as the ellipse $E_{1}$ is (by assumption) defined by its two conjugate radii $O P$ and $O R$. Let also $S_{y}$ be the $y$-shear with the same shearing vector $\mathbf{u}$ which (similarly to $S_{x}$ ) transforms point $P \in C$ also into $R$. Indeed, from (2.4) and setting again $u:=|\mathbf{u}|=r / \rho$, we obtain $S_{y}(P)=R$. Also $S_{y}(Q)=Q$ (as the vertical axis $y^{\prime} O y$ is invariant under $S_{y}$ ), and hence $Q \in C \cap E_{2}$. Thus $S_{y}(C)=E_{2}$ as the ellipse $E_{2}$ (by assumption) is defined by its two conjugate radii $O Q$ and $O R$. From the above discussion, point $R$ is an intersecting point of the ellipses $E_{1}$ and $E_{2}$, i.e. $R \in E_{1} \cap E_{2}$.

The given pairs of line segments $(O P, O R)$ and $(O Q, O R)$ are indeed correspond to pairs of conjugate radii for $E_{1}$ and $E_{2}$ respectively, as these segments are affine transformations (recall $S_{x}$ and $S_{y}$ ) of the $C^{\prime}$ s orthogonal radii $O P$ and $O Q$. As affinity preserves parallelism, the tangent lines of $E_{1}$ and $E_{2}$ at their points $P$ and $Q$ respectively are parallel to $O R$, while the tangent lines of $E_{1}$ and $E_{2}$ at their point $R$ are parallel to $O P$ and $O Q$ respectively (because the tangent lines of the circle $C$ at its points $P$ and $Q$ are, trivially, parallel to its orthogonal radii $O Q$ and $O P$ ). Figure 4 clarifies also the above discussion (on which the referred ellipses $E_{x}$ and $E_{y}$ correspond to $E_{1}$ and $E_{2}$ respectively) as the square frame around $C$ is transformed, through $S_{x}$ and $S_{y}$, into the parallelograms around $E_{1}$ and $E_{2}$.

Lemma 3.2 can now be applied, where we have to replace $E_{x}$ and $E_{y}$ with $E_{1}$ and $E_{2}$ respectively and set $u:=r / \rho$. Therefore, a c.t.e. $E$ of $E_{1}, E_{2}$ and $C$ always exists with directive angle $\omega$ (in the adopted o.c.s.), and hence point $R$ lie onto $E^{\prime}$ s major semi-axis. Moreover, through Lemma 3.2, the foci separation of the c.t.e. $E$ is then $f=2 u \rho$, i.e. $f=2 r=2|O R|$ (as $u:=r / \rho$ ). Thus, the intersection point $R \in E_{1} \cap E_{2}$ is indeed a focal point of the common tangential $E$. Moreover, the $E^{\prime}$ s principal radii are then given by $a=\rho \sqrt{u^{2}+1}=\sqrt{\rho^{2}+r^{2}}$ and $b=\rho$, while its eccentricity $\varepsilon:=a^{-1} \sqrt{a^{2}-b^{2}}=u / \sqrt{u^{2}+1}=r / \sqrt{\rho^{2}+r^{2}}$. The c.t.e. $E$ can then be analytically expressed (in the adopted o.c.s.) as (3.15), where we set $u:=r / \rho$, and therefore (3.27) holds.

For the calculations of the two contact points $T_{1}\left(x_{1}, y_{1}\right)$ and $T_{1}^{\prime}\left(-x_{1},-y_{1}\right)$ between $E_{1}$ and its tangential ellipse $E$, as well as for their corresponding tangent lines $t_{1}$ and $t_{1}^{\prime}$ (at $T_{1}$ and $T_{1}^{\prime}$ ), we consider the following approach. The analytical expression of $E_{1}$ is given by (2.13), as $E_{1}=S_{x}(C)$, where we set $u:=r / \rho$. Let now $x=x_{0}$ be a vertical tangent line of $E_{1}$ at some point $T_{0}\left(x_{0}, y_{0}\right) \in E_{1}, y_{0}>0$. Then, setting $x:=x_{0}$ into (2.13), a trinomial with respect to $y$ is derived, say $\eta_{1}=\eta_{1}\left(y ; x_{0}\right)=0$. Because we expect $\eta_{1}(y)=0$ to have one real (double) root $y=y_{0}$ (due to the fact that line $x=x_{0}$ was assumed to be a tangent line of $E_{1}$ at $T_{0}$ ), its discriminant must be zero, i.e. $4 u^{2} \sin ^{2} \omega\left(u^{2} \rho^{2} \cos ^{2} \omega+\rho^{2}-x_{0}^{2}\right)=0$, or equivalently

$$
x_{0}= \pm \rho \sqrt{1+u^{2} \cos ^{2} \omega}= \pm \sqrt{\rho^{2}+r^{2} \cos ^{2} \omega},
$$

while setting the $x_{0}$ value, as above, to the trinomial $\eta\left(y ; x_{0}\right)=0$, its double real roots would then given by

$$
y_{0}= \pm \frac{u^{2} \rho \sin 2 \omega}{2 \sqrt{1+u^{2} \cos ^{2} \omega}}=\frac{1}{2} x_{0}^{-1} r^{2} \sin 2 \omega .
$$

Adopting the positive value for $x_{0}$, from the above relation we obtain

$$
x_{0}=\sqrt{\rho^{2}+r^{2} \cos ^{2} \omega} \text { and } y_{0}=\frac{r^{2} \sin 2 \omega}{2 \sqrt{\rho^{2}+r^{2} \cos ^{2} \omega}} .
$$

Similarly, if $x=x_{0}^{\prime}$ is now assumed to be also a vertical tangent line of $E$ at some point $T_{0}^{\prime}\left(x_{0}^{\prime}, y_{0}^{\prime}\right) \in E$, then by setting $x:=x_{0}^{\prime}$ into (3.27), a trinomial with respect also to $y$ is derived, say $\eta_{1}^{\prime}=\eta_{1}^{\prime}\left(y ; x_{0}^{\prime}\right)=0$. As we expect $\eta_{1}^{\prime}(y)=0$ to have also one real (double) root $y=y_{0}^{\prime}$ (due to the fact that line $x=x_{0}^{\prime}$ was assumed to be a tangent line of $E$ at $T_{0}^{\prime}$ ), its discriminant must again be zero. The new calculations yields that $x_{0}^{\prime}$ and $y_{0}^{\prime}$ expressions are 
exactly the same as $x_{0}$ and $y_{0}$ in (3.32), i.e. $x_{0}^{\prime}=x_{0}$ and $y_{0}^{\prime}=y_{0}$. Therefore, the vertical tangent lines of $E_{1}$ and $E$ are coincide at point $T_{0}=T_{0}^{\prime}$, i.e. the contact point $T_{1}\left(x_{1}, y_{1}\right)$ of $E_{1}$ and $E$ is indeed given by (3.32), where $x_{0}$ and $y_{0}$ notations were replaced by $x_{1}$ and $y_{1}$ respectively, and hence (3.28) holds. The corresponding tangent line $t_{1}$ at point $T_{1}$ is thus the vertical tangent line $x=x_{1}\left(=x_{0}=x_{0}^{\prime}\right)$ which is proved to be a common tangent line between $E_{1}$ and $E$. For clarification see also Fig. 5.

For the two contact points $T_{2}\left(x_{2}, y_{2}\right), y_{2}>0$, and $T_{2}^{\prime}\left(-x_{2},-y_{2}\right)$ between $E_{2}$ and $E$, as well as for their corresponding tangent lines $t_{2}$ and $t_{2}^{\prime}\left(\right.$ at $T_{2}$ and $T_{2}^{\prime}$ ), we consider the analytical expression of $E_{2}$ given in (2.5), as $E_{2}=S_{y}(C)$, where we also have to set $u:=r / \rho$. Let $y=y_{0}$ be a horizontal tangent line of $E_{2}$ at some point $S_{0}\left(\chi_{0}, \psi_{0}\right) \in E_{2}, \psi_{0}>0$. Then, by setting $y:=\psi_{0}$ into (2.5), a trinomial with respect to $x$ is derived, say $\eta_{2}=\eta_{2}\left(x ; \psi_{0}\right)=0$. Because we expect $\eta_{2}(x)=0$ to have one real (double) root $x=\chi_{0}$ (due to the fact that line $y=\psi_{0}$ was assumed to be a tangent line of $E_{2}$ at $S_{0}$ ), its discriminant must be zero, i.e. $4 u^{2} \cos ^{2} \omega\left(u^{2} \rho^{2} \sin ^{2} \omega+\rho^{2}-y_{0}^{2}\right)=0$, or equivalently

$$
\psi_{0}= \pm \rho \sqrt{1+u^{2} \sin ^{2} \omega}= \pm \sqrt{\rho^{2}+r^{2} \sin ^{2} \omega},
$$

while adopting the positive value for $\psi_{0}$ as above, and setting it to the trinomial $\eta_{2}\left(x ; \psi_{0}\right)=0$, its double real root is then given by

$$
\chi_{0}=\frac{u^{2} \rho \sin 2 \omega}{2 \sqrt{1+u^{2} \sin ^{2} \omega}}=\frac{r^{2} \sin 2 \omega}{2 \sqrt{\rho^{2}+r^{2} \sin ^{2} \omega}} .
$$

Similarly, if $y=\psi_{0}^{\prime}$ is assumed to be a horizontal tangent line of $E$ at some point $S_{0}^{\prime}\left(\chi_{0}^{\prime}, \psi_{0}^{\prime}\right) \in E, \psi_{0}^{\prime}>0$ then, by setting $y:=\psi_{0}^{\prime}$ into (3.27), a trinomial with respect also to $x$ is derived, say $\eta_{2}^{\prime}=\eta_{2}^{\prime}\left(x ; \psi_{0}^{\prime}\right)=0$. As we expect the trinomial $\eta_{2}^{\prime}(x)=0$ to have one real (double) root $x=\chi_{0}^{\prime}$ (due to the fact that line $y=\psi_{0}^{\prime}$ was assumed to be a tangent line of $E$ at $S_{0}^{\prime}$ ), its discriminant must again be zero. The calculations yield that the values of $\chi_{0}^{\prime}$ and $\psi_{0}^{\prime}$ are coincide to the $\chi_{0}$ and $\psi_{0}$ values as in (3.34), i.e. $\chi_{0}^{\prime}=\chi_{0}$ and $\psi_{0}^{\prime}=\psi_{0}$. Therefore, the horizontal tangent lines of $E_{2}$ and $E$ are coincide at point $S_{0}=S_{0}^{\prime}$, i.e. the contact point $T_{2}\left(x_{2}, y_{2}\right), y_{2}>0$, between $E_{2}$ and $E$ is indeed $S_{0}\left(\chi_{0}, \psi_{0}\right)$, and hence (3.29) holds. The corresponding tangent line $t_{2}$ at point $T_{2}$ is then coincide with the horizontal tangent line $y=y_{2}\left(=\psi_{0}=\psi_{0}^{\prime}\right)$ which is proved to be a common tangent line of $E_{2}$ and $E$. For clarification see also Fig. 5.

For the last two contact points $T_{3}\left(x_{3}, y_{3}\right)$ and $T_{3}^{\prime}\left(-x_{3},-y_{3}\right)$ between circle $C$ and its tangential ellipse $E$, it holds that $x_{3}^{2}+y_{3}^{2}=\rho^{2}$. Moreover, the slope of diameter $T_{3} T_{3}^{\prime}$ is given by $\tan (\omega+\pi / 2)=y_{3} / x_{3}$. This is due to the fact that ellipse $E$ is a tangential ellipse to $C$. Hence, the minor axis of $E$ (orthogonal to the $E^{\prime}$ s major axis which forms an angle $\omega$ with o.c.s.'s horizontal axis) is assumed to be equal to the radius of $C$, i.e. $\rho=b$; see also Fig. 5 . The above two relations imply that

$$
x_{3}=-y_{3} \tan \omega \text { and } y_{3}^{2}=\frac{\rho^{2}}{1+\tan ^{2} \omega}=\rho^{2} \cos ^{2} \omega,
$$

and therefore (3.30) is derived. The corresponding two tangent lines $t_{3}$ and $t_{3}^{\prime}$ of $C$ at their contact points $T_{3}$ and $T_{3}^{\prime}$ respectively, are given in their usual forms $t_{3}: x_{3} x+y_{3} y=\rho^{2}$ and $t_{3}^{\prime}: x_{3} x+y_{3} y=-\rho^{2}$ (on the adopted o.c.s.), as they are tangent lines of the circle $C$, and hence by substitution of (3.30) into them, relations (3.31) hold. Therefore, the tangent lines $t_{3}$ and $t_{3}^{\prime}$ are parallel to the non-orthogonal radii $O R$, as their slopes are both $\tan \omega$.

Example 3.1. Consider the bundle of three line segments $O P, O Q$ and $O R$ as in Example 2.1, i.e. $\rho=|O P|=$ $|O Q|:=10$ and $r=|O R|:=15$, with $O P \perp O Q$ and $\omega=\measuredangle(O P, O R):=\pi / 6\left(=30^{\circ}\right)$. These three given line segments correspond to three mutually conjugate radii which define three mutually conjugate ellipses such that $E_{1}=E_{1}(O P, O R), E_{2}=E_{2}(O Q, O R)$ and $E_{3}=E_{3}(O P, O Q)$; see also Fig. 5. Notably, $E_{3}$ is a circle of radius $\rho=10$ as defined by the pair $(O P, O Q)$ of its orthogonal (and equal) conjugate radii. Consider also point $O$ as the origin of an o.c.s. with its horizontal and vertical axes spanned, respectively, by orthogonal radii $O P$ and $O Q$.

Ellipses $E_{1}$ and $E_{2}$ can be expressed as the $x$ - and $y$-shears of circle $E_{3}$, with shearing vector $\mathbf{u}:=\rho^{-1} O R$ of length $u:=r / \rho=1.5$. Therefore, $E_{1}$ and $E_{2}$ are then given in Example 2.1, where $E_{x}$ and $E_{y}$ correspond now to $E_{1}$ and $E_{2}$ respectively, while their principal radii $0<b_{i}<a_{i}, i=1,2$, and their directive angles $\theta_{i}, i=1,2$, are given respectively by the values of $a_{x}, b_{x}, a_{y}, b_{y}$ and $\theta_{x}, \theta_{y}$ in Example 2.1. Applying now Theorem 3.1, a c.t.e. $E$ of the given three mutually conjugate ellipses $E_{i}, i=1,2,3$, always exists, and its major axis is spanned by (nonorthogonal common radius) $O R$, while point $R$ is being one of its foci. The corresponding $E$ 's principal radii are 
then given by $a=\sqrt{\rho^{2}+r^{2}}=5 \sqrt{13} \approx 18.0278$ and $b=\rho=10$, while eccentricity, foci distance, and surface area are given, respectively, by $\varepsilon=\frac{3}{13} \sqrt{13} \approx 0.832, f=30$ and $A=50 \pi \sqrt{13} \approx 566.359$. As $E^{\prime}$ 's focal point, say $F$, is identical to $R$, then $F=R(r \cos \omega, r \sin \omega)=R\left(\frac{15}{2} \sqrt{3}, \frac{15}{2}\right)$ and $F^{\prime}\left(-\frac{15}{2} \sqrt{3},-\frac{15}{2}\right)$ are then correspond to the two diametrical foci of $E$. The analytical expression of c.t.e. $E$ is given, through (3.27), by $E: 25 x^{2}-18 \sqrt{3} x y+43 y^{2}=5200$.

The two contact points $T_{1}\left(x_{1}, y_{1}\right), y_{1}>0$, and $T_{1}^{\prime}\left(-x_{1},-y_{1}\right)$ between ellipse $E_{1}$ and its tangential $E$, are calculated through (3.28), i.e. $x_{1}=\frac{5}{2} \sqrt{43} \approx 16.394$ and $y_{1}=\frac{45}{86} \sqrt{129} \approx 5.943$, with their corresponding two tangent lines $t_{1}$ and $t_{1}^{\prime}$ (at their contact points $T_{1}$ and $T_{1}^{\prime}$ ) to be parallel to $O Q$ (which spans o.c.s.'s vertical axis $\left.y^{\prime} O y\right)$, i.e. $t_{1}: x=x_{1}=\frac{5}{2} \sqrt{43}$ and $t_{1}^{\prime}: x=-x_{1}=-\frac{5}{2} \sqrt{43}$.

The other two contact points $T_{2}\left(x_{2}, y_{2}\right), y_{2}>0$, and $T_{2}^{\prime}\left(-x_{2},-y_{2}\right)$ between ellipse $E_{2}$ and its tangential $E$, are calculated through (3.29), i.e. $x_{2}=\frac{9}{2} \sqrt{13} \approx 7.7942$ and $y_{2}=25 / 2=12.5$, with their corresponding two tangent lines $t_{2}$ and $t_{2}^{\prime}$ (at their contact points $T_{2}$ and $T_{2}^{\prime}$ ) to be parallel to $O P$ (which spans o.c.s.'s horizontal axis $x^{\prime} O x$ ), i.e. $t_{2}: y=y_{2}=25 / 2$ and $t_{2}^{\prime}: y=-y_{2}=-25 / 2$.

Finally, the last two contact points $T_{3}\left(x_{3}, y_{3}\right), y_{3}>0$, and $T_{3}^{\prime}\left(-x_{3},-y_{3}\right)$ between circle $E_{3}$ and its tangential $E$, are calculated through (3.30), i.e. $x_{3}=-5$ and $y_{3}=5 \sqrt{3} \approx 8.6603$, with their corresponding two tangent lines $t_{3}$ and $t_{3}^{\prime}$ (at their contact points $T_{3}$ and $T_{3}^{\prime}$ ) obtained through (3.30), i.e. $t_{3}: \sqrt{3} y-x=20$ and $t_{3}^{\prime}: x-\sqrt{3} y=20$, which are parallel to the non-orthogonal radii $O R$, as their slopes are both $\tan (\pi / 6)=\sqrt{3} / 3$.

Figure 5 visualizes exactly Example 3.1 by presenting the three mutually conjugate ellipses $E_{1}=E_{1}(O P, O R)$, $E_{2}=E_{2}(O Q, O R)$ and $E_{3}=E_{3}(O P, O Q)$, with their defining three given mutually conjugate radii as well as their foci points. The c.t.e. $E$ of $E_{i}, i=1,2,3$, is also presented, together with their six common tangent lines at their six corresponding contact points. The intersection angles of the ellipses $E_{i}, i=1,2,3$, at their common points $P, Q$ and $R$, are also denoted, while $\vartheta:=\pi / 2-\omega$ and $\varphi:=\measuredangle(O P, O Q)=\pi / 2$.

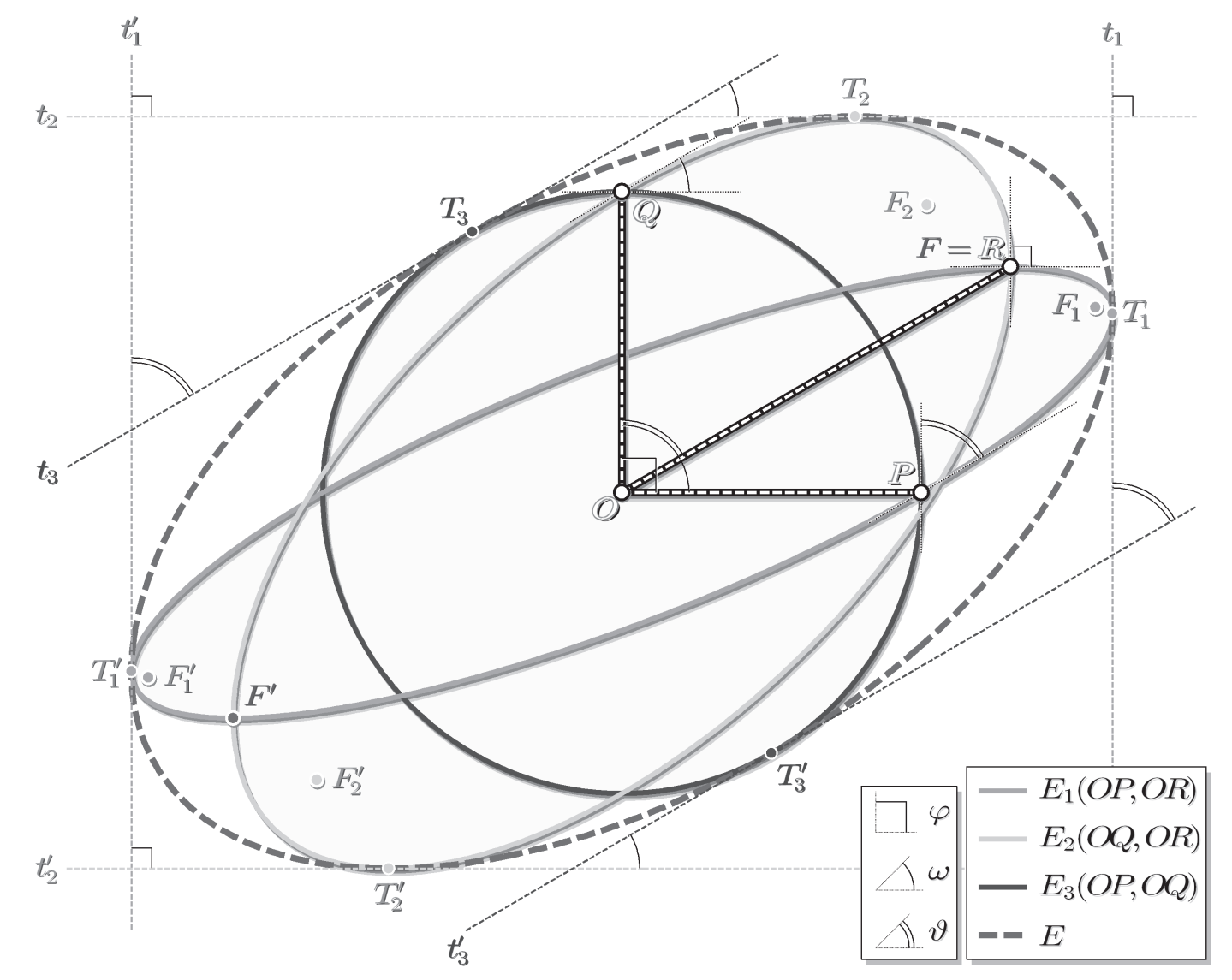

Figure 5. Graphs of the three mutually conjugate ellipses $E_{i}, i=1,2,3$, of Example 3.1, and their c.t.e. E together with their common tangent lines.

Recall Lemma 3.2 where a c.t.e. $E$ of $E_{x}, E_{y}$ and $C$ was obtained. In the following, the number of all existing common tangential ellipses (like $E$ ) of the ellipses $E_{x}, E_{y}$ and $C$ is investigated. 
Lemma 3.3. Let $C$ be a circle of radius $\rho>0$, centered at the origin $O$ of an o.c.s., while $E_{x}$ and $E_{y}$ are the $x$ - and $y$-shears of $C$ as in Lemma 3.2. There are, at most, two distinct common tangential ellipses, say $E$ and $E^{*}$, of $E_{x}, E_{y}$ and $C$, where $E$ is given in (3.27) having directive angle $\omega$ (i.e. its major axis is spanned by the shearing vector of length $u$ that transforms $C$ into $E_{x}$ and $E_{y}$ ), while the second c.t.e. $E^{*}(\neq E)$ have (when it exists) a directive angle $\theta^{*}$ (with respect to $O P$ ) such that

$$
\frac{\tan \theta^{*}}{\tan \omega}=-\frac{1+u^{2} \cos 2 \omega}{1-u^{2} \cos 2 \omega}=\frac{u^{2}+1-\left(u^{2}-1\right) \tan ^{2} \omega}{u^{2}-1-\left(u^{2}+1\right) \tan ^{2} \omega} .
$$

When $\omega \in(0, \pi / 2)$ the second c.t.e. $E^{*}$ (of $E_{x}, E_{y}$ and $C$ ) exists if and only if $\omega \in\left(0, \omega_{0}\right)$ where

$$
\omega_{0}:= \begin{cases}\frac{1}{2} \arccos \sqrt{2 u^{-2}-u^{-4}}, & \text { if } u \geq \sqrt{2} / 2, \\ \pi / 2, & \text { if } u<\sqrt{2} / 2 .\end{cases}
$$

Proof. Consider an o.c.s. with origin at the center $O$ of the given circle $C$. We assume that $E^{*}$ is a c.t.e., other than $E$, i.e. an ellipse centered at $O$ which is also (simultaneously) tangent to $E_{x}, E_{y}$ and $C$. Its principal radii are denoted with $a^{*}>b^{*}:=\rho$ and its directive angle with $\theta^{*}$. Therefore, it holds that $E^{*}=E_{x}^{*}=E_{y}^{*}$, where $E_{x}^{*}$ and $E_{y}^{*}$ denote the two tangential ellipses of $E_{x}$ and $E_{y}$ respectively in the direction of $\theta^{*}$ and have principal radii $a_{x}>b_{x}:=\rho$ and $a_{y}>b_{y}:=\rho$.

The aspect ratio of the tangential ellipse $E_{x}^{*}\left(=E^{*}\right)$ of $E_{x}$, can be obtained through the angle, say $\theta$, between (the major axes of) $E_{x}$ and its tangential $E_{x}^{*}$, i.e. $\theta:=\theta^{*}-\theta_{x}$, as we did similarly in the proof of Lemma 3.2 (where had set $\theta:=\omega-\theta_{x}$ ). Firstly, the relation (2.15) can be expressed in terms of $\delta_{x}$, as in (3.19). In particular, from (3.19), it holds that

$$
\cos ^{2} \omega=\frac{1}{2}(1+\cos 2 \omega)=\frac{1}{4} u^{-2}\left(\delta_{x}-2\right)\left(\delta_{x}-2 u^{2}\right),
$$

as $0<\delta_{x}<2$ and $\delta_{x}<2 u^{2}$, and applying (3.38) into (2.15), we obtain

$$
\tan \theta_{x}=\sqrt{\frac{\delta_{x}\left(2 u^{2}-\delta_{x}\right)}{\left(2-\delta_{x}\right)\left(2 u^{2}+2-\delta_{x}\right)}}
$$

as also $\delta_{x}<2\left(u^{2}+1\right)$. Substituting then (3.39) into

$$
\cos ^{2} \theta=\frac{1}{1+\tan ^{2}\left(\theta^{*}-\theta_{x}\right)}=\frac{\left(1+\tan \theta^{*} \tan \theta_{x}\right)^{2}}{\left(1+\tan \theta^{*} \tan \theta_{x}\right)^{2}+\left(\tan \theta^{*}-\tan \theta_{x}\right)^{2}},
$$

we obtain (after some algebra) that

$$
\cos \theta=\frac{\sqrt{\delta_{x}\left(2 u^{2}-\delta_{x}\right)} \sin \theta^{*}+\sqrt{\left(2-\delta_{x}\right)\left(2 u^{2}+2-\delta_{x}\right)} \cos \theta^{*}}{2 \sqrt{2 u^{2}+2-\delta_{x}}} .
$$

The aspect ratio $\lambda_{x}^{*}$ of $E_{x}^{*}$ can then be obtained from (3.20) where $\theta:=\theta^{*}-\theta_{x}$. In particular, applying (3.23) into (3.20), it holds that

$$
\lambda_{x}^{* 2}=\frac{2 \delta_{x}\left[1-u^{2}+\left(u^{2}+1-\delta_{x}\right)\left(1-2 \cos ^{2} \theta\right)\right]}{\left(\delta_{x}-2 \cos ^{2} \theta\right)\left[4 u^{2}-\left(\delta_{x}-2\right)\left(\delta_{x}-2 u^{2}\right)\right]+2 \delta_{x}^{2} \sin \theta},
$$

and then, through (3.40), we derive that

$$
\lambda_{x}^{* 2}=\frac{2\left(1-u^{2}\right)+\left[\delta_{x}\left(\delta_{x}-2 u^{2}-2\right)+2\left(u^{2}+1\right)\right] \cos 2 \theta^{*}+\eta_{x} \sin 2 \theta^{*}}{2\left[\delta_{x}\left(\delta_{x}-2 u^{2}-2\right)+2\left(u^{2}+1\right)\right] \cos ^{2} \theta^{*}+\eta_{x} \sin 2 \theta^{*}},
$$

where $\eta_{x}:=\sqrt{\delta_{x}\left(2-\delta_{x}\right)\left(2 u^{2}-\delta_{x}\right)\left(2 u^{2}+2-\delta_{x}\right)}$ or, by substitution of $\delta_{x}$ as in (3.19), $\eta_{x}=u^{2} \sqrt{2(1-\cos 4 \omega)}=$ $u^{2}|\sin 2 \omega|$.

Similarly to the case of the tangential ellipse $E_{x}^{*}$ as above, we now consider as angle $\theta$ between (the major axes of) $E_{y}$ and $E_{y}^{*}$, the difference $\theta:=\theta^{*}-\theta_{y}$. In particular, from (3.26), it holds

$$
\cos ^{2} \omega=\frac{1}{2}(1+\cos 2 \omega)=\frac{1}{4} u^{-2} \delta_{y}\left(2 u^{2}+2-\delta_{y}\right)
$$


as it is easy to see, through (3.26), that $0<\delta_{y}<2\left(u^{2}+1\right)$. Applying (3.42) into (2.7), we obtain

$$
\tan \theta_{y}=\sqrt{\frac{\left(2-\delta_{y}\right)\left(2 u^{2}+2-\delta_{y}\right)}{\delta_{y}\left(2 u^{2}-\delta_{y}\right)}}
$$

as $0<\delta_{y}<2$ and $\delta_{y}<2 u^{2}$ also hold. Substituting then (3.43) into

$$
\cos ^{2} \theta=\frac{1}{1+\tan ^{2}\left(\theta^{*}-\theta_{y}\right)}=\frac{\left(1+\tan \theta^{*} \tan \theta_{y}\right)^{2}}{\left(1+\tan \theta^{*} \tan \theta_{y}\right)^{2}+\left(\tan \theta^{*}-\tan \theta_{y}\right)^{2}},
$$

we obtain (after some algebra) that

$$
\cos \theta=\frac{\sqrt{\left(2-\delta_{y}\right)\left(2 u^{2}+2-\delta_{y}\right) \sin \theta^{*}}+\sqrt{\delta_{y}\left(2 u^{2}-\delta_{y}\right)} \cos \theta^{*}}{2 \sqrt{u^{2}+1-\delta_{y}}} .
$$

The aspect ratio $\lambda_{y}^{*}$ of $E_{y}^{*}$ can then be obtained from (3.25) where $\theta:=\theta^{*}-\theta_{y}$. In particular, applying firstly (3.42) into (3.25), it holds that

$$
\lambda_{y}^{* 2}=\frac{u^{2}-1+\left(u^{2}+1-\delta_{y}\right)\left(1-2 \cos ^{2} \theta\right)}{2\left(\delta_{y}-u^{2}-1\right) \cos ^{2} \theta+\delta_{y}\left(2 u^{2}+2-\delta_{y}\right)},
$$

and then, through (3.44), we derive that

$$
\lambda_{y}^{* 2}=-\frac{2\left(u^{2}-1\right)+\left[\delta_{y}\left(\delta_{y}-2 u^{2}-2\right)+2\left(u^{2}+1\right)\right] \cos 2 \theta^{*}-\eta_{y} \sin 2 \theta^{*}}{2\left[\delta_{y}\left(\delta_{y}-2 u^{2}-2\right)+2\left(u^{2}+1\right)\right] \sin ^{2} \theta^{*}+\eta_{y} \sin 2 \theta^{*}},
$$

where $\eta_{y}:=\sqrt{\delta_{y}\left(2-\delta_{y}\right)\left(2 u^{2}-\delta_{y}\right)\left(2 u^{2}+2-\delta_{y}\right)}$.

We assumed earlier that $E^{*}=E_{x}^{*}=E_{y}^{*}$ so that ellipse $E^{*}$ can be another c.t.e. (besides $E$ ) of $E_{x}, E_{y}$ and $C$. Thus, the aspect ratios in (3.41) and (3.45) must coincide to the $E^{* \prime}$ s aspect ratio $\lambda^{*}:=\lambda_{x}=\lambda_{y}$, as the minor radii of $E_{x}^{*}$ and $E_{y}^{*}$ must be equal with $C^{\prime}$ s radius $\rho$ as assumed, i.e. as $b_{x}=b_{y}=\rho$. Writing the equation $\lambda_{x}{ }^{2}-\lambda_{y}{ }^{2}=0$ in terms of $\tan \theta^{*}$, through (3.41) and (3.45), we obtain the trinomial expression

$$
\mathrm{Atan}^{2} \theta^{*}+\mathrm{B} \tan \theta^{*}+\Gamma=0
$$

where the coefficients $\mathrm{A}, \mathrm{B}$ and $\Gamma$ are given by

$$
\begin{aligned}
& \mathrm{A}:=-\left(\delta_{x}-2\right)\left(\delta_{x}-2 u^{2}\right)\left[\delta_{y}\left(\delta_{y}-2 u^{2}-2\right)+2\left(u^{2}+1\right)\right], \\
& \mathrm{B}:=2 \eta_{x}\left[4 u^{2}-\delta_{y}\left(2 u^{2}+2-\delta_{x}\right)\right]-\eta_{y}\left[4 u^{2}-\delta_{x}\left(2 u^{2}+2-\delta_{x}\right)\right], \\
& \Gamma:=\left(\delta_{y}-2\right)\left(\delta_{y}-2 u^{2}\right)\left[\delta_{x}\left(\delta_{x}-2 u^{2}-2\right)+2\left(u^{2}+1\right)\right] .
\end{aligned}
$$

Notice that the trinomial with respect to $\tan \theta^{*}$ (3.46) has two, at most, real roots and one of which is expected always to be $\tan \omega$, because in the direction of angle $\omega$ the c.t.e. $E$ (of $E_{x}, E_{y}$ and $C$ ), as in (3.27), always exists (recall Lemma 3.2) with aspect ratio $\lambda=\sqrt{u^{2}+1}$. Therefore, assuming that there exists another tangential ellipse $E^{*} \neq E$ (of $E_{x}, E_{y}$ and $C$ ), the trinomial (3.46) implies that

$$
\begin{gathered}
\tan \omega=\frac{1}{2 \mathrm{~A}}\left(-\mathrm{B} \pm \sqrt{\mathrm{B}^{2}-4 \mathrm{~A} \Gamma}\right), \tan \theta^{*}=\frac{1}{2 \mathrm{~A}}\left(-\mathrm{B} \mp \sqrt{\mathrm{B}^{2}-4 \mathrm{~A} \Gamma}\right) \text { or } \\
\tan \theta^{*}=-\frac{\mathrm{B}}{\mathrm{A}}-\tan \omega .
\end{gathered}
$$

By setting $\theta^{*}:=\omega$ into the trinomial (3.46), it holds that $-\mathrm{B} \tan \omega=\Gamma+\mathrm{A} \tan ^{2} \omega$, and then using of (3.48) we obtain an alternative form of (3.48), i.e.

$$
\tan \theta^{*} \tan \omega=\frac{\Gamma}{\mathrm{A}}
$$

Moreover, the coefficients $\mathrm{A}$ and $\Gamma$, as in (3.47a) and (3.47c), can be simplified in the forms of

$$
\mathrm{A}=-8 u^{2}\left(1-u^{2} \cos 2 \omega\right) \cos ^{2} \omega \text { and } \Gamma=8 u^{2}\left(1+u^{2} \cos 2 \omega\right) \sin ^{2} \omega,
$$


by substitution of $\delta_{x}$ as in (3.19). Relation (3.49) finally yields (3.36), through (3.50).

When $E^{*}$ is an ellipse (of direction angle $\theta^{*} \neq \omega$ ), the value $\lambda^{*}:=\lambda_{x}$ would then correspond to aspect ratio of ellipse $E^{*}$, and hence $\mathbb{R}_{+} \ni \lambda^{*} \leq 1$, while the value $a^{*}:=a_{x}=b_{x} / \lambda_{x}=\rho / \lambda^{*}$ provides $E^{* \prime}$ major radius (as $\rho$ is always assumed to be $E^{* \prime}$ s minor radius). Recall that always $a_{x} \geq b_{x}$ when the values $a_{x}$ and $b_{x}$ are real and given by (2.14). Hence, the real-valued ratio $\lambda_{x}:=b_{x} / a_{x}$ cannot be greater than 1 or, equivalently, the ratio in (3.41) cannot be $>1$. However, if the (real-valued) ratio as in (3.41) is found to be negative, i.e. $\lambda^{* 2}\left(:=\lambda_{x}^{2}\right)<0$, then $a^{* 2}=\rho^{2} / \lambda^{* 2}<0$, which is equivalent to the fact that the $E^{*}$ is shaped now by a hyperbola $E^{*}:(\tilde{y} / \rho)^{2}-\left(\tilde{x} /\left|a^{*}\right|\right)^{2}=1, \tilde{x}, \tilde{y} \in \mathbb{R}$ (in some o.c.s.) instead of an ellipse (tangential to $E_{x}, E_{y}$ and $C$ ).

Therefore, $E^{*}$ is indeed a c.t.e. of $E_{x}, E_{y}$ and $C$, if and only if (iff) the ratio in (3.41) is equal or lower than 1 or, equivalently, rewriting (3.41) in terms of $\tan \theta^{*}$, iff

$$
\lambda^{* 2}=\frac{4-\delta_{x}\left(2 u^{2}+2-\delta_{x}\right)+2 \eta_{x} \tan \theta^{*}-\left(2-\delta_{x}\right)\left(2 u^{2}-\delta_{x}\right) \tan ^{2} \theta^{*}}{2 \delta_{x}\left(\delta_{x}-2 u^{2}-2\right)+4\left(u^{2}+1\right)+2 \eta_{x} \tan \theta^{*}} \leq 1 .
$$

By substitution of $\eta_{x}=u^{2}|\sin 2 \omega|$ (shown earlier) and $\delta_{x}$, as in (3.19), the above condition is equivalent to

$$
\frac{1-u^{2}+2 u^{2} \cos ^{2} \omega+u^{2}|\sin 2 \omega| \tan \theta^{*}}{1-u^{2}+u^{2} \cos ^{2} \omega+u^{2}|\sin 2 \omega| \tan \theta^{*}-u^{2} \cos ^{2} \omega \tan ^{2} \theta^{*}} \geq 1 .
$$

Otherwise, if the ratio as in (3.51) is negative, $E^{*}$ is then being a hyperbola which is (simultaneously) tangent and concentric to $E_{x}, E_{y}$ and $C$.

The iff condition (3.51) can be simplified. In particular, when the denominator of the ratio in (3.51) is assumed to be negative, then (3.51) implies that $u^{2} \cos ^{2} \omega\left(1+\tan ^{2} \theta^{*}\right) \leq 0$, which cannot hold, and thus the denominator in (3.51) must always be positive. Therefore the condition (3.51) is equivalent to

$$
u^{2} \cos ^{2} \omega \tan ^{2} \theta^{*}-(\operatorname{sgn} \omega)(\operatorname{sgn} \cos \omega) u^{2} \sin 2 \omega \tan \theta^{*}+u^{2} \sin ^{2} \omega-1<0,
$$

where $\operatorname{sgn}(\cdot)$ denotes the usual sign operator, or using (3.36),

$$
\left\{2 s\left[\left(1+u^{2}\right)^{2}-4 u^{4} \cos ^{4} \omega\right]+\left(1+u^{2}+2 u^{2} \cos ^{2} \omega\right)^{2}\right\} u^{2} \sin ^{2} \omega-\left(1-u^{2} \sin ^{2} \omega\right)\left(1+u^{2}-2 u^{2} \cos ^{2} \omega\right)^{2}<0,
$$

where $s:=(\operatorname{sgn} \omega) \operatorname{sgn}\left(\frac{\pi}{2}-|\omega|\right)$. When $\omega \in(0, \pi / 2)$, the condition (3.52) is then reduced, as $s=1$, to

$$
1-2 u^{2}+u^{4} \cos ^{2} 2 \omega>0,
$$

which is equivalent to $\omega \in\left(0, \omega_{0}\right)$, with $\omega_{0}$ as in the first branch of (3.37), provided that $u \geq \sqrt{2} / 2$. However, if $u<\sqrt{2} / 2$ is assumed, the condition (3.52) always holds for any angle such that $s=1$. Hence $\omega \in(0, \pi / 2)$.

Using the above Lemma 3.3 it can now be shown that, under certain conditions, a second c.t.e. of three mutually conjugate ellipses, with one of them being a circle, also exists.

Theorem 3.2. Consider a circle $C$ of radius $\rho>0$ centered at $O$, and two ellipses $E_{1}$ and $E_{2}$, all three being mutually conjugate, i.e. $E_{1}, E_{2}$ and $C$ are defined by a bundle of three given line segments (corresponding th their mutually conjugate radii) $O P, O Q$ and $O R$, such that $E_{1}=E_{1}(O P, O R), E_{2}=E_{2}(O Q, O R)$ and $C=C(O P, O Q)$. Let $r:=|O R|$ and $\omega:=\angle(O P, O R)$. Then, at most, two common tangential ellipses of $E_{1}, E_{2}$ and $C$ can exist. That is, besides the always existing c.t.e. $E$ (which its major axis is spanned by the non-orthogonal segment $O R$ of the bundle, recall Theorem 3.1), a second c.t.e. $E^{*}(\neq E)$ can also exist, and when it does its major axis forms an angle $\theta^{*}$ with $O P$ (i.e. $\theta^{*}$ is the directive angle of $E^{*}$ with respect to $O P$ ), and is given by

$$
\frac{\tan \theta^{*}}{\tan \omega}=-\frac{\rho^{2}+r^{2} \cos 2 \omega}{\rho^{2}-r^{2} \cos 2 \omega}=-\frac{\rho^{2}+r^{2}+\left(\rho^{2}-r^{2}\right) \tan ^{2} \omega}{\rho^{2}-r^{2}+\left(\rho^{2}+r^{2}\right) \tan ^{2} \omega},
$$

The second c.t.e. $E^{*}$ (of $E_{1}, E_{2}$ and $C$ ) with $\omega \in(0, \pi / 2)$, exists if and only if $\omega \in\left(0, \omega_{0}\right)$ where

$$
\omega_{0}:= \begin{cases}\frac{1}{2} \arccos \left(\rho r^{-2} \sqrt{2 r^{2}-\rho^{2}}\right), & \text { if } r \geq \rho \sqrt{2} / 2, \\ \pi / 2, & \text { if } r<\rho \sqrt{2} / 2 .\end{cases}
$$

www.iejgeo.com 
Equivalently assumed that $\omega \in(0, \pi / 2)$, the second c.t.e. $E^{*}$ exists if and only if $r / \rho \in\left(0, u_{-}\right) \cup\left(u_{+},+\infty\right)$, where

$$
u_{ \pm}:=(\sec 2 \omega) \sqrt{1 \pm \sin 2 \omega} .
$$

The principal radii $0<b^{*}<a^{*}$ of $E^{*}$ are then given by

$$
a^{*}=\sqrt{\frac{\left(\rho^{2}-r^{2}\right)\left(\rho^{4}-r^{4} \cos ^{2} 2 \omega\right)}{\left(\rho^{2}-r^{2} \cos 2 \omega\right)^{2}-4 \rho^{2} r^{2} \sin ^{2} \omega}} \text { and } b^{*}=\rho,
$$

while $E^{*}$ is analytically expressed, on an o.c.s. spanned by orthogonal radii $O P$ and $O Q$, as

$$
\begin{aligned}
E^{*}: & {\left[a^{* 2}\left(\rho^{2}+r^{2} \cos 2 \omega\right)^{2} \tan ^{2} \omega-\rho^{2}\left(\rho^{2}-r^{2} \cos 2 \omega\right)^{2}\right] x^{2}+\left[a^{* 2}\left(\rho^{2}-r^{2} \cos 2 \omega\right)^{2}+\rho^{2}\left(\rho^{2}+r^{2} \cos 2 \omega\right)^{2} \tan ^{2} \omega\right] y^{2}+} \\
& 2\left(\rho^{2}-a^{* 2}\right)\left(\rho^{4}-r^{4} \cos ^{2} 2 \omega\right)(\tan \omega) x y=\rho^{2} a^{* 2}\left[\left(\rho^{2}-r^{2} \cos 2 \omega\right)^{2}+\left(\rho^{2}+r^{2} \cos 2 \omega\right)^{2} \tan ^{2} \omega\right] .
\end{aligned}
$$

Proof. Note that $|O P|=|O Q|=\rho$ and $O P \perp O Q$ as $C$ is a circle of radius $\rho>0$. Consider an o.c.s. with origin at $C$ 's center $O$, with its horizontal and vertical axes spanned by the orthogonal radii $O P$ and $O Q$ respectively. Let $\mathscr{B}=\left\{\mathbf{e}_{1}, \mathbf{e}_{2}\right\}$ be the o.c.s.'s orthonormal vector base.

Similar to the (beginning of the) proof of Theorem 3.1, by setting $u:=r / \rho$ the ellipses $E_{x}$ and $E_{y}$ of Lemma 3.3 are now correspond to the ellipses $E_{1}$ and $E_{2}$, while the line segments $O P, O Q$ and $O R$, indeed, define the three mutually conjugate ellipses as $E_{1}=E_{1}(O P, O R), E_{2}=E_{2}(O Q, O R)$ and $C=C(O P, O Q)$, and hence being three mutually conjugate radii of $E_{1}, E_{2}$ and $C$. Moreover, Lemma 3.3 implies that, at most, two common tangential ellipses of $E_{1}, E_{2}$ and $C$ can exist. Notably, a c.t.e. $E$ in the direction of $O R$, always exists and it is analytically expressed (in the adopted o.c.s.) by (3.27), while a second c.t.e. $E^{*}(\neq E)$, can also exist if and only if $\omega=\measuredangle(O P, O R) \in\left(0, \omega_{0}\right)$, with $\omega_{0}$ as in (3.55), as directly derived form Lemma 3.3 by setting $u:=r / \rho$.

Recalling the inequality (3.53), the second c.t.e. $E^{*}(\neq E)$ can also exist, assumed that $\omega \in(0, \pi / 2)$, when $1-2 u^{2}+$ $u^{4} \cos ^{2} 2 \omega>0$, which is equivalent (by solving the inequality with respect to $\left.u\right)$ to $r / \rho=u \in\left(0, u_{-}\right) \cup\left(u_{+},+\infty\right)$ with $u_{ \pm}$as in (3.56).

The major radius $a^{*}$ of the second c.t.e. $E^{*}$ (when $E^{*}$ exists) is then given by $a^{*}=b^{*} / \lambda^{*}=\rho / \lambda^{*}$ (as its minor radius $\left.b^{*}=\rho\right)$, where the $E^{* \prime}$ s aspect ratio $\lambda^{*}\left(:=\lambda_{x}=\lambda_{y}\right)$ is obtained through $\left(\lambda^{*}\right)^{-2}=\ell$ where $\ell$ being the ratio in (3.51). By substitution of $\tan \theta^{*}$, as in (3.54), into (3.51) we have

$$
\lambda^{* 2}=\frac{\left(u^{2} \cos 2 \omega-1\right)^{2}-4 u^{2} \sin ^{2} \omega}{\left(u^{2}-1\right)\left(u^{4} \cos ^{2} 2 \omega-1\right)},
$$

and by setting $u:=r / \rho$, the major radius $a^{*}=\rho / \lambda^{*}$ as in (3.57) is obtained. Moreover, $E^{*}$ can then be analytically expressed (in the orthonormal vector base $\mathscr{B}$ ) by (2.1), where we must set $\omega:=\theta^{*}, a:=a^{*}, b:=b^{*}=\rho$ and $u:=r / \rho$. Hence, (3.58) is finally derived by the substitution of $\theta^{*}$ as in (3.54).

The following Example demonstrates a case of non-existent second c.t.e. of three mutually conjugate ellipses with one of them is being a circle. A common tangential hyperbola is calculated instead.

Example 3.2. Recall the bundle of the three given line segments $O P, O Q$ and $O R$, as given in Example 3.1, which define the three mutually conjugate ellipses $E_{1}=E_{1}(O P, O R), E_{2}=E_{2}(O Q, O R)$ and $E_{3}=E_{3}(O P, O Q)$, with $E_{3}$ being a circle of radius $\rho>0$. Then, a c.t.e. $E$ of these ellipses exists in the direction of the nonorthogonal segment $O R$ having point $R$ as one of its foci; see also Fig. 5. According to Theorem 3.2, a second c.t.e. $E^{*}(\neq E)$ of $E_{i}, i=1,2,3$, can exist iff $\omega \in\left(0, \omega_{0}\right)$. However, this is not the case here. Indeed, calculating $\theta^{*}$ as in (3.54) and $\omega_{0}$ as in (3.55), it holds that $\pi / 2>\pi / 6=: \omega=\measuredangle(O P, O R)>\omega_{0}=\frac{1}{2} \arccos \left(\frac{2}{9} \sqrt{14}\right) \approx 16.8744^{\circ}$ (which cannot hold), while $15=: r>\rho / \sqrt{2}=5 \sqrt{2} \approx 7.0711$, i.e. $30^{\circ}=: \omega \notin\left(0, \omega_{0}\right) \approx\left(0,16.8744^{\circ}\right)$. Therefore, the ellipse $E$ is the only c.t.e. of $E_{i}, i=1,2,3$, that exists. Moreover, the analytical expression (in the adopted o.c.s.) of $E^{*}$, as in (3.58), implies, through $\rho:=10$ and $r:=15$, that $E^{*}:-313 x^{2}+306 \sqrt{3} x y+226 y^{2}+34000=0$, which is clearly a hyperbola as it is expected. This is because the above condition of $\omega$ (suggested by Theorem 3.2) does not fulfilled, as $30^{\circ} \notin\left(0,16.8744^{\circ}\right)$, which means that the ratio in (3.51) must be < 1, i.e. $E^{* \prime}$ s aspect ratio $\lambda^{*} \in \mathbb{C}$. Indeed, the above can be confirmed calculating the values of $a^{*}$ and $b^{*}$, as in (3.57), i.e. $a^{*}=\frac{5}{143} \sqrt{12155 i} \approx 3.8549 \mathrm{i}$ and $b^{*}=\rho=10$. From the above expression for $E^{*}$ we conclude that the canonical form of the hyperbola $E^{*}$ can be written as $E^{*}:(\hat{x} / \rho)^{2}-\left(\hat{y} /\left|a^{*}\right|\right)^{2}=1, \hat{x}, \hat{y} \in \mathbb{R}$, as $0<\left|a^{*}\right|<\rho$, and hence the (major) semi-axis length of hyperbola $E^{*}$ (which is the minimum distance of $E^{*}$ from its center) is then $\rho$ while 
its imaginary (or minor) semi-axis length is then $\left|a^{*}\right|=\frac{5}{143} \sqrt{12155}$. The directive angle of the hyperbola $E^{*}$ with respect to $O P$ (i.e. the angle of its (major) axis with respect to the horizontal axis of the adopted o.c.s.) is then given by $\theta^{*}-\pi / 2$ where $\theta^{*}$ as in (3.54), i.e. $\theta^{*}=\arctan \left(\frac{17}{3} \sqrt{3}\right)-\frac{\pi}{2} \approx-5.8175^{\circ}$. This is so, because in case $E^{*}$ was an ellipse, the value of $\tan \theta^{*}$ given in (3.54) corresponds to the slope of $E^{* \prime} \mathrm{s}$ major axis, while in the present hyperbola case, the value of $\tan \theta^{*}$ corresponds to the slope of $E^{* \prime}$ imaginary (minor) axis, as $E^{*}$ is now expressed by $E^{*}:-\left(\tilde{x} /\left|a^{*}\right|\right)^{2}+(\tilde{y} / \rho)^{2}=1$ where $0<\left|a^{*}\right|<\rho$. Thus, due to the orthogonality of the principal axes, we have to subtract $\pi / 2$ from the value of $\theta^{*}$ as in (3.54).

Figure 6 visualizes exactly Example 3.2, by presenting the three mutually conjugate ellipses $E_{1}=E_{1}(O P, O R)$, $E_{2}=E_{2}(O Q, O R)$ and $E_{3}=E_{3}(O P, O Q)$ as well as their c.t.e. $E$, exactly as in Fig. 5, together with their common tangential (c.t.) hyperbola $E^{*}$ of $E_{i}, i=1,2,3$.

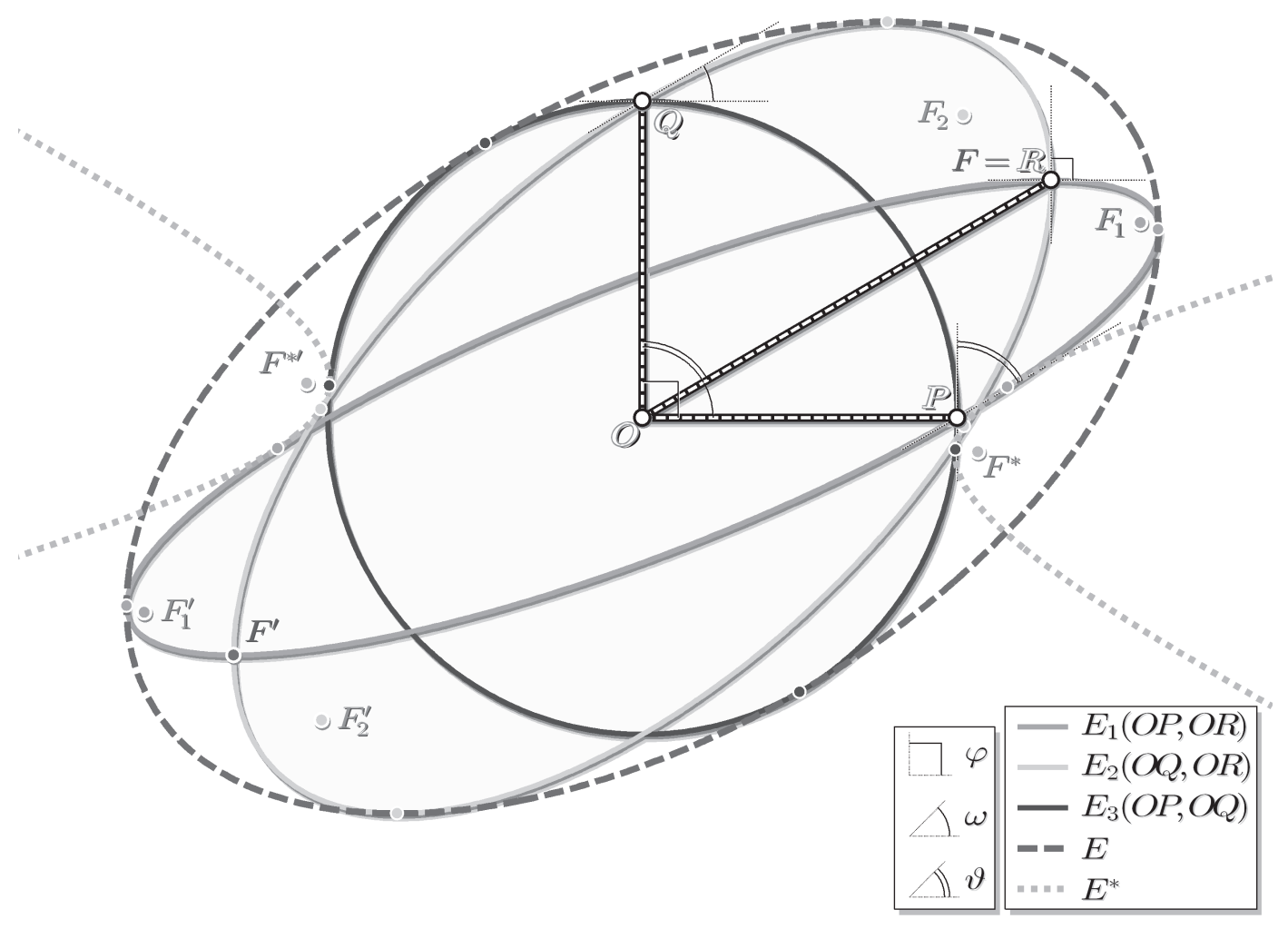

Figure 6. Graphs of the three mutually conjugate ellipses $E_{i}, i=1,2,3$, as in Example 3.2, together with their c.t. ellipse $E$ and c.t. hyperbola $E^{*}$

The following Example demonstrates the existence of a second c.t.e. of three mutually conjugate ellipses with one of them being a circle.

Example 3.3. Consider a bundle of three given line segments $O P, O Q$ and $O R$ as in Example 3.1, or 3.2, where this time $O R^{\prime}$ s length is set to be the one-third of the $O P^{\prime}$ s length, i.e. $r=|O R|:=|O P| / 3=5$. Recall from Example 3.1 that $\rho=|O P|=|O Q|:=10$ with $O P \perp O Q$ and $\omega=\measuredangle(O P, O R):=\pi / 6\left(=30^{\circ}\right)$. These line segments are considered to be the three mutually conjugate radii that define three mutually conjugate ellipses, i.e. $E_{1}=E_{1}(O P, O R), E_{2}=E_{2}(O Q, O R)$ and $E_{3}=E_{3}(O P, O Q)$. Note that $E_{3}$ is a circle of radius $\rho>0$. Consider also (as in Example 3.1) an o.c.s. spanned by the orthogonal radii $O P$ and $O Q$. Similarly to the Example 2.1, the analytical expressions of the ellipses $E_{1}$ and $E_{2}$ are then given by (2.13) and (2.5) respectively, where $S_{x}(C)$ and $S_{y}(C)$ correspond now to $E_{1}$ and $E_{2}$ respectively, with $u:=r / \rho=1 / 2$, i.e. $E_{1}: x^{2}-2 \sqrt{3} x y+19 y^{2}=100$ and $E_{2}: 17 x^{2}-2 \sqrt{3} x y+3 y^{2}=300$, while $E_{3}$ corresponds to the circle $E_{3}: x^{2}+y^{2}=100$. A c.t.e. $E$ of $E_{i}, i=1,2,3$, exists and its major axis is spanned by the non-orthogonal radius $O R$, while point $R$ being one of its foci; see Fig. 7 . The analytical expression (on the adopted o.c.s.) is given, through (3.27), by $E: 17 x^{2}-2 \sqrt{3} x y+19 y^{2}=2000$. As far as the additional c.t.e. of $E_{i}, i=1,2,3$, is concerned, it holds that $1 / 2=r / \rho \in\left(0, u_{-}\right) \approx(0,0.732)$, as $\pi / 6=: \omega \in(0, \pi / 2)$. Therefore, from Theorem 3.2, we conclude that (unlike Example 3.2) there exist two, in total, common tangential 
ellipses, $E$ and $E^{*}$, of the three mutually conjugate ellipses $E_{i}, i=1,2,3$. The existing second c.t.e. $E^{*}$ has then principal radii $0<b^{*}<a^{*}$ and directive angle $\theta^{*}$ given, through (3.57) and (3.54), by $a^{*}=\frac{5}{11} \sqrt{77} \approx 11.9659$, $b^{*}=10$ and $\theta^{*}=-\arctan \left(\frac{3}{7} \sqrt{3}\right) \approx-36.5868^{\circ}$, while, through (3.58), its analytical expression is of the form $E^{*}: 203 x^{2}+42 \sqrt{3} x y+225 y^{2}=25200$.

The following Fig. 7 is an exact visualization of Example 3.3, by presenting the three mutually conjugate ellipses $E_{1}=E_{1}(O P, O R), E_{2}=E_{2}(O Q, O R)$ and $E_{3}=E_{3}(O P, O Q)$ together with their foci, as well as their two (in total) common tangential ellipses $E$ and $E^{*}$. The intersection angles between the ellipses $E_{i}, i=1,2,3$ at their points $P, Q$ and $R$ are also depicted.

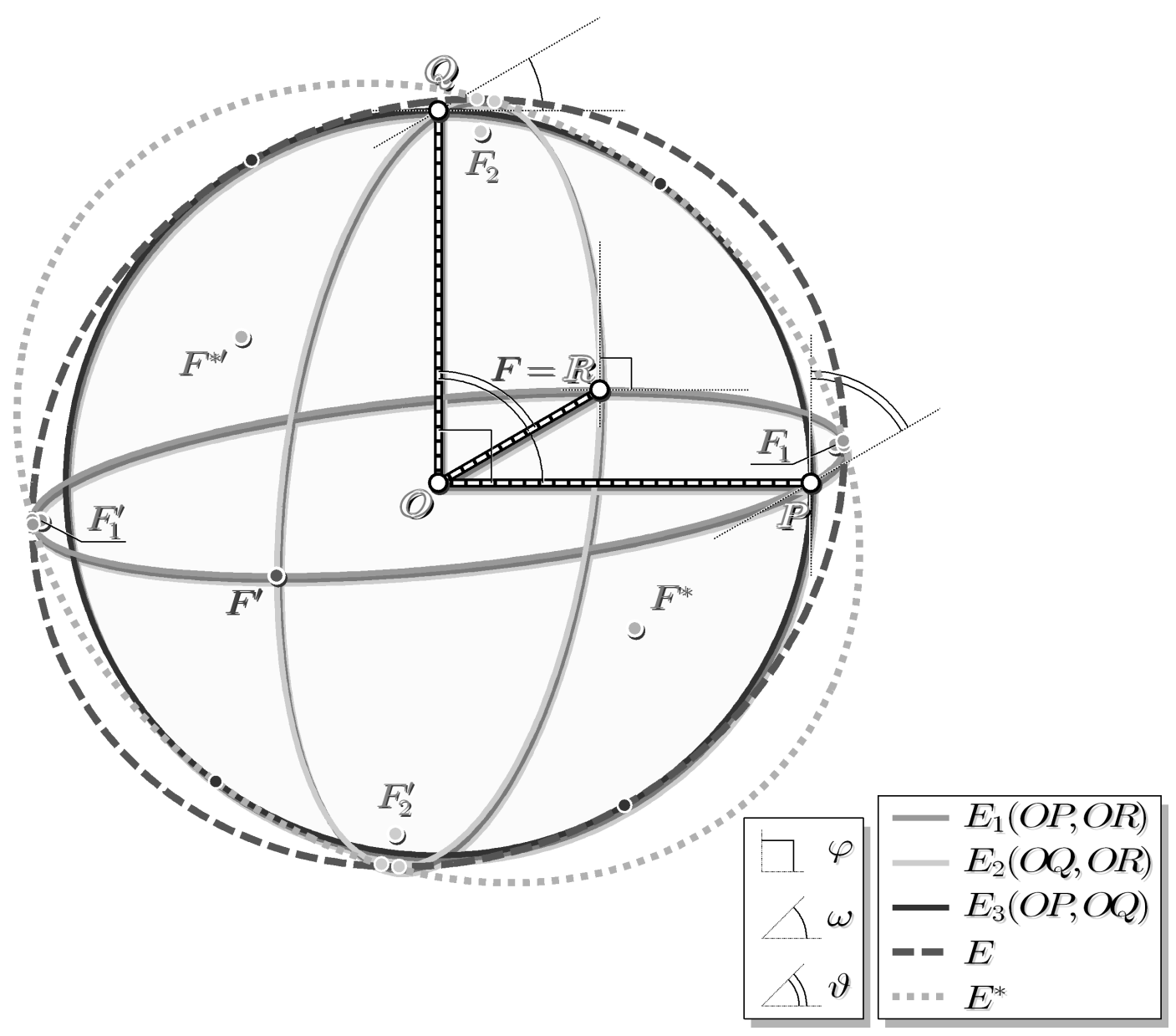

Figure 7. Graphs of the three mutually conjugate ellipses $E_{i}, i=1,2,3$, as in Example 3.3, together with their two common tangential ellipses $E$ and $E^{*}$

\subsection{The general case}

We shall now extend the results of the orthogonal case, as discussed in sub-Section 3.1, to the general case of three mutually conjugate ellipses (including the case where none of them is a circle).

From the orthogonal case, investigated in Theorem 3.1, we concluded that there always exists a c.t.e. around three given mutually conjugate ellipses, when one of them is a circle. The tangential ellipse $E$ can then be considered as a "primary" c.t.e., while any other c.t.e. that might exist can be considered as a "secondary" c.t.e. (of $E_{i}, i=1,2,3$ ). Having the above in mind, we consider the following definitions.

Definition 3.2. Let $E_{i}, i=1,2,3$, be three mutually conjugate ellipses. We can always consider a coordinate system in which one of the ellipses is expressed as a circle, and hence two of their three mutually conjugate radii, which define $E_{i}, i=1,2,3$, are of equal length and orthogonal with each other. A c.t.e. $E$ of $E_{i}, i=1,2,3$, shall then be called primary when its major semi-axis coincides with the non-orthogonal radius from the corresponding three mutually conjugate radii (defining $E_{i}, i=1,2,3$ ).

Definition 3.3. Any other non-primary c.t.e. of $E_{i}, i=1,2,3$, shall be called as a secondary c.t.e. of $E_{i}, i=1,2,3$. 
Before we proceed with the main Theorem, a special cyclic sum notation $\mathfrak{S}$ is introduced here. In particular, the following form of "multiple" cyclic sums shall be used, i.e.

$$
\bigcup_{\substack{p, q, r \\ \varphi, \vartheta, \omega}} f(p, q, r ; \varphi, \vartheta, \omega):=f(p, q, r ; \varphi, \vartheta, \omega)+f(q, r, p ; \vartheta, \omega, \varphi)+f(r, p, q ; \omega, \varphi, \vartheta) .
$$

The cyclic sum as above refers to a three-termed sum of the variable-depending expression $f$, where the summation is simultaneously cycle through the three values $p, q, r$ and $\varphi, \vartheta, \omega$, i.e. according to the scheme of indexes' sequence: $\begin{gathered}p \rightarrow q \rightarrow r \rightarrow p \rightarrow \cdots \\ p \rightarrow \vartheta \rightarrow \omega \rightarrow \varphi \rightarrow \cdots\end{gathered}$

The following main Theorem investigates the existence of common tangential ellipses around three mutually conjugate ellipses, and therefore extends Peschka's Proposition 1.1. It also provides a plane-geometric proof of it.

Main Theorem. Consider three mutually conjugate ellipses $E_{i}, i=1,2,3$, defined by a bundle of three given line segments which correspond to $E_{i}$ 's three mutually conjugate radii. These given radii, say $O P, O Q$ and $O R$, are determined by their lengths $p, q, r>0$ respectively, and the two angles $\varphi:=\measuredangle(O P, O Q) \in(0, \pi)$ and $\omega:=\measuredangle(O P, O R) \in(0, \pi)$. Let also $\vartheta:=\angle(O R, O Q) \in(0, \pi)=\varphi-\omega$. Then, there always exists a primary c.t.e. $E$ of $E_{i}$, $i=1,2,3$, with their corresponding common tangent lines, between each $E_{i}, i=1,2,3$, and their c.t.e. $E$, being parallel to each of the three given conjugate radii. Moreover, a unique secondary c.t.e. $E^{*}(\neq E)$ of $E_{i}, i=1,2,3$, with $\varphi>\omega$, can exist if and only if

$$
\bigcup_{\substack{p, q, r \\ \varphi, \vartheta, \omega}} p^{4} q^{2} \sin ^{2} \varphi\left(q^{2} \sin ^{2} \varphi-2 r^{2} \sin ^{2} \omega\right)>0,
$$

or when it holds

$$
2 r^{2}\left(p^{2} \sin ^{2} \omega+q^{2} \sin ^{2} \vartheta\right)<p^{2} q^{2} \sin ^{2} \varphi .
$$

In case one of the mutually conjugate ellipses is reduced to a circle (and therefore two out of three $E_{i}$ 's mutually conjugate radii are orthogonal and have equal length), then the foci of the primary c.t.e. $E$ coincide with the end points of the non-orthogonal diameter of the three mutually conjugate diameters (spanned by the three given mutually conjugate radii) which define $E_{i}, i=1,2,3$.

Proof. Consider an o.c.s. where its horizontal and vertical axes are spanned by radii $O P$ and $O Q^{\prime}$ respectively, where $O Q^{\prime} \perp O P$ and $\left|O Q^{\prime}\right|=|O P|$. Hence, the corresponding orthonormal vector base is given by $\mathscr{B}:=\left\{\mathbf{e}_{1}:=\right.$ $\left.O P /|O P|, \mathbf{e}_{2}:=O Q^{\prime}|| O Q^{\prime} \mid\right\}$, i.e. $P_{\mathscr{B}}(p, 0)$ and $Q_{\mathscr{B}}^{\prime}(0, p)$, or simply $P(p, 0)$ and $Q^{\prime}(0, p)$. For point $Q$ it then holds that $Q(q \cos \varphi, q \sin \varphi)$. Let also $E_{1}:=E_{1}(O P, O R), E_{2}:=E_{2}(O Q, O R)$ and $E_{3}:=E_{3}(O P, O Q)$ be three mutually conjugate ellipses defined by their corresponding three mutually conjugate radii $O P, O Q$ and $O R$. As pointed out in Definition 3.2, a new c.s. can be adopted so that one of the ellipses $E_{i}$ can be expressed as a circle. Without loss of generality, we may choose the c.s. in which ellipse $E_{3}$ is expressed as circle $C(O, p)$. It then holds that $O P \perp O Q$ and $|O P|=|O Q|$, i.e. $\varphi=\pi / 2$ and $p=q$. Hence, Theorem 3.1 can be applied in order to derive the primary c.t.e. $E$ of $E_{i}, i=1,2,3$. Moreover, due to the fact that $0<\vartheta=\varphi-\omega$, or $\omega<\varphi$, the given radius $O R$ lies "between" $O P$ and $O Q$, as $\omega \in(0, \varphi) \subset(0, \pi)$; see also Fig. 5 where $O R$ lies between $O P$ and $O Q$. Therefore, Theorem 3.2 can also be applied in order to derive the secondary (if any) c.t.e $E^{*}$ of $E_{i}, i=1,2,3$.

For the construction of this new c.s., in which $E_{3}$ corresponds to circle $C(O, p)$, we consider the following: Let $C$ be a circle of radius $p$ centered at $O$, and its points $P(p, 0)$ and $Q^{\prime}(0, p)$. Let $S_{x}: \mathbf{x}^{\prime}=\mathbf{N}_{\mathbf{u}} \mathbf{x}$ be a $x-$ shear transformation with shearing vector $\mathbf{u}:=p^{-1} O Q$ in the initially adopted o.c.s. (of vector base $\mathscr{B}$ ). Then, $S_{x}$ transforms point the $Q^{\prime}(0, p)$ into $Q(q \cos \varphi, q \sin \varphi)$. Indeed, substituting the shearing vectors' length $u:=|\mathbf{u}|=q / p$ and its angle $\omega:=\varphi$ into (2.3), the $x$-shear transformation matrix is then given by

$$
\mathbf{N}_{\mathbf{u}}=\left(\begin{array}{cc}
1 & u \cos \omega \\
0 & u \sin \omega
\end{array}\right)=\left(\begin{array}{cc}
1 & \frac{q}{p} \cos \varphi \\
0 & \frac{q}{p} \sin \varphi
\end{array}\right)
$$

and hence it can be easily verified that $S_{x}\left(Q^{\prime}\right)=Q$. Moreover, it holds that $S_{x}(P)=P$, as the horizontal axis $x^{\prime} O x$ (in $\mathscr{B}$ ) is an $S_{x}$-invariant. Therefore, the $x$-sheared circle $C$, i.e. $S_{x}(C)$, is essentially the ellipse $E_{3}$, as the orthogonal pair of radii $\left(O P, O Q^{\prime}\right)$ is transformed into the pair of conjugate radii $(O P, O Q)$ which define ellipse $E_{3}$, as $E_{3}=E_{3}(O P, O Q)$ is assumed, i.e. $S_{x}(C)=E_{3}$ with $P \in E_{3} \cap E_{1}$ and $Q \in E_{3} \cap E_{2}$.

Consider now a new vector base, say $\mathscr{B}^{\prime}$, in which ellipse $E_{3}$ is expressed as circle $C(O, p)$ or, equivalently, $O P \perp_{\mathscr{B}^{\prime}} O Q$ and $|O P|_{\mathscr{B}^{\prime}}=|O Q|_{\mathscr{B}^{\prime}}$. Essentially, the affine transformation $S_{x}$ (as defined above) corresponds to a 
vector base change, from $\mathscr{B}$ to $\mathscr{B}^{\prime}$, of the form $\mathbf{x}=\mathbf{N}_{\mathbf{u}} \mathbf{x}^{\prime}$, in which $P_{\mathscr{B}^{\prime}}(p, 0)$ and $Q_{\mathscr{B}^{\prime}}(0, p)$. The length $r^{\prime}:=|O R|_{\mathscr{B}}$ and angle $\omega^{\prime}:=\measuredangle_{\mathscr{B}^{\prime}}(O P, O R)$ can now be calculated in order to use them instead of values $r:=|O P|$ and $\omega:=\measuredangle(O P, O R)$, for the application of Theorems 3.1 and 3.2. The components' vector $\mathbf{r}$ of $O R$ is also given, in the initial vector base $\mathscr{B}$, by $\mathbf{r}=\left(r_{1}, r_{2}\right)^{\mathrm{T}}:=(r \cos \omega, r \sin \omega)^{\mathrm{T}} \in \mathbb{R}^{2 \times 1}$. When $O R$ is expressed in $\mathscr{B}^{\prime}$, its new components' vector, say $\mathbf{r}^{\prime}$, is then given by $\mathbf{r}^{\prime}=\left(r_{1}^{\prime}, r_{2}^{\prime}\right)^{\mathrm{T}}:=\left(r^{\prime} \cos \omega^{\prime}, r^{\prime} \sin \omega^{\prime}\right)^{\mathrm{T}} \in \mathbb{R}^{2 \times 1}$. Applying the vector base change $\mathbf{x}=\mathbf{N}_{\mathbf{u}} \mathbf{x}^{\prime}$ on the vector $\mathbf{r}$, we obtain that $\mathbf{r}=\mathbf{N}_{\mathbf{u}} \mathbf{r}^{\prime}$, or

$$
\left(r_{1}^{\prime}, r_{2}^{\prime}\right)^{\mathrm{T}}=\mathbf{r}^{\prime}=\mathbf{N}_{\mathbf{u}}^{-1} \mathbf{r}=r(\csc \varphi)\left(\sin (\varphi-\omega), \frac{p}{q} \sin \omega\right)^{\mathrm{T}},
$$

and thus $r^{\prime}=|O R|_{\mathscr{B}^{\prime}}=\left|\mathbf{r}^{\prime}\right|_{\mathscr{B}^{\prime}}=\sqrt{r_{1}^{\prime 2}+r_{2}^{\prime 2}}$, while $O R^{\prime}$ s corresponding angle $\omega^{\prime}$ is then given by $\omega^{\prime}=\measuredangle_{\mathscr{B}^{\prime}}(O P, O R)=$ $\arctan \left(r_{2}^{\prime} / r_{1}^{\prime}\right) \in(-\pi, \pi)$. Therefore, from (3.63), we derive after some algebra, that

$$
r^{\prime}=\frac{r}{q}(\csc \varphi) \sqrt{p^{2} \sin ^{2} \omega+q^{2} \sin ^{2} \vartheta} \text { and } \omega^{\prime}=\arctan \left(\frac{p \sin \omega}{q \sin \vartheta}\right) .
$$

Recall the mutually conjugate ellipses $E_{1}=E_{1}(O P, O R), E_{2}=E_{2}(O Q, O R)$ and $E_{3}=E_{3}(O P, O Q)$, with $P_{\mathscr{B}^{\prime}}(p, 0), Q_{\mathscr{B}^{\prime}}(0, p)$ and $R_{\mathscr{B}^{\prime}}\left(r_{1}^{\prime}, r_{2}^{\prime}\right)$. Therefore, in vector base $\mathscr{B}^{\prime}$, where the ellipse $E_{3}$ corresponds to circle $C(O, \rho)$, Theorem 3.1 can be applied, and hence the primary c.t.e. $E$ of $E_{i}, i=1,2,3$ (as in Definition 3.2), always exists. Recalling Theorem 3.1, the non-orthogonal radii $O R$ coincides with $E^{\prime}$ 's major semi-axis, while the foci of $E$ are essentially the end points of (non-orthogonal) diameter spanned by radius $O R$. In vector base $\mathscr{B}^{\prime}$, Theorem 3.1 shows also that each pair of (diametrical) common tangent lines $\left(t_{i}, t_{i}^{\prime}\right)$ between ellipse $E_{i}$ and its primary c.t.e. $E$, for $i=1,2,3$, are being parallel to each of the three given mutually conjugate radii $O P, O Q$ and $O R$. In particular, $t_{1}$ and $t_{1}^{\prime}$ are parallel to $O Q, t_{2}$ and $t_{2}^{\prime}$ are parallel to $O P$, while $t_{3}$ and $t_{3}^{\prime}$ are parallel to $O R$. Therefore, as $S_{x}$ preserves parallelism, we conclude that also in the initially adopted vector base $\mathscr{B}$ the common tangent lines between each $E_{i}$ and $E$ are parallel to each (of the three) mutually conjugate radii that define $E_{i}, i=1,2,3$.

Moreover, according to Theorem 3.2 and Definition 3.3, a secondary c.t.e. $E^{*}$ of $E_{i}, i=1,2,3$, exists under certain conditions. This secondary tangential $E^{*}$-when exists - is also unique, i.e. it is the only non-primary c.t.e. of $E_{i}, i=1,2,3$. Recall that a second tangential $E^{*}(\neq E)$ can exist, iff (3.53) holds, i.e. iff

$$
p^{4}-2 p^{2}{r^{\prime}}^{2}+r^{\prime 4} \cos ^{2} 2 \omega^{\prime}>0,
$$

where we have set $u:=r^{\prime} / p$ and $\omega:=\omega^{\prime}$ (as values $r$ and $\omega$ in (3.53) correspond now to values $r^{\prime}$ and $\omega^{\prime}$ in vector base $\mathscr{B}^{\prime}$ ). Thus, applying (3.64) into (3.65) we derive (after some algebra) the equivalent condition

$$
p^{4} q^{2} \sin ^{2} \varphi\left(2 r^{2} \sin ^{2} \omega-q^{2} \sin ^{2} \varphi\right)+q^{4} r^{2} \sin ^{2} \vartheta\left(2 p^{2} \sin ^{2} \varphi-r^{2} \sin ^{2} \vartheta\right)+r^{4} p^{2} \sin ^{2} \omega\left(2 q^{2} \sin ^{2} \vartheta-p^{2} \sin ^{2} \omega\right)<0,
$$

which can be written in the compact cyclic sum form of (3.60).

Inequality (3.65) holds trivially for every $\omega^{\prime} \in(0, \pi)$ if $r^{\prime}<p / \sqrt{2}$, i.e. if $p^{4}-2 p^{2} r^{\prime 2}>0$. That is, when $p>\sqrt{2} r^{\prime}$ or, equivalently through (3.64), when (3.61) holds, then general condition (iff) (3.65) also holds for every $\omega^{\prime} \in(0, \pi)$. Therefore, the unique secondary tangential ellipse $E^{*}$ also exists when (3.61) is assumed.

The following Example shows the use of conditions (3.60) and (3.61) for concluding the existence or not of a secondary c.t.e. $E^{*}$.

Example 3.4. Recall Example 3.1, where it was shown that there is only one c.t.e. of three mutually conjugate ellipses $E_{i}, i=1,2,3$, i.e. the primary c.t.e. $E$ (as expected). Setting now $\varphi=\measuredangle(O P, O Q):=\pi / 2$ into the special (if) condition (3.61), as well as $p=q:=\rho=10, r:=15$ and $\omega=\measuredangle(O P, O R):=\pi / 6$, we derive that (3.61) yields $-35000>0$ which cannot hold. As the condition in (3.61) is not an "if and only if" condition, we cannot conclude the non-existence of a secondary c.t.e. $E^{*}$. Moreover, by substitution of the above values into the condition as in (3.60), we derive that $223437500<0$ which also cannot hold, but this time we can safely conclude that the second c.t.e. does not exist, as (3.60) is an iff condition. The special condition (3.61) can, however, determine the existence of the second c.t.e. $E^{*}$ of $E_{i}, i=1,2,3$, as in Example 3.3. Indeed, setting $\varphi:=\pi / 2, \omega:=\pi / 6, p=q:=10$ and $r:=5$ into (3.61), it is obtained that $5000>0$ which holds, and hence $E^{*}$ exists. This also can be confirmed by calculating the general (iff) condition (3.60) which then implies that $-5156250<0$ which also holds.

Finally, we point out that the analytical expressions of the two common tangential ellipses $E$ and $E^{*}$, can be calculated (in the vector base $\mathscr{B}^{\prime}$ ) by setting $r:=r^{\prime}$ and $\omega:=\omega^{\prime}$ both into (3.27) and (3.58) respectively, where $r^{\prime}$ and $\omega^{\prime}$ are as in (3.64). 


\section{Discussion}

Consider the problem of finding a concentric common tangent ellipse around three given coplanar and concentric ellipses, say $E_{i}, i=1,2,3$, each one defined by a pair of two conjugate semi-diameters which are taken from a bundle of three given line segments. This plane-geometric problem was first stated and addressed by G. A. Peschka in [5], in his proof of Pohlke's Fundamental Theorem of Axonometry. However, his proof is based on a parallel projection of an appropriate sphere $S$ onto $E_{i}$ 's common plane, say $\mathbb{E}$. Indeed, these mutually conjugate ellipses $E_{i}, i=1,2,3$, correspond to the parallel projections of three maximum circles of the sphere $S$ (lying on three planes orthogonal with each other). According to [4,5], the common tangential ellipse, say $E$, of all $E_{i}, i=1,2,3$, is then the parallel projection of the sphere's contour onto $\mathbb{E}$. This parallel projection method has been used in literature for the proof of Pohlke's Fundamental Theorem of Axonometry.

With this present paper, the above "Peschka's problem", which is a plane-geometric problem, is addressed exclusively in terms of Analytic Plane Geometry, while it was also thoroughly investigated. Moreover, not only did it was found that a common tangent ellipse (around three given mutually conjugate ellipses, as $E_{i}$, $i=1,2,3$ ) always exists, but also found all the existed concentric and tangent ellipses around three given mutually conjugate ellipses. It was proved that there exist two, in total, c.t.e.: the primary one $E$ (which corresponds to the parallel-projected contour of sphere $S$ as above), and the secondary one $E^{*}(\neq E)$ which exists under certain conditions. When the secondary c.t.e. $E^{*}$ does not exist, then a (concentric to $E_{i}$ ) hyperbola exists instead, with its two branches being tangent to all three given mutually conjugate ellipses $E_{i}, i=1,2,3$. The provided examples and figures were demonstrating these results.

\section{References}

[1] Emch, A., Proof of Pohlke's theorem and its generalizations by affinity. American Journal of Mathematics 40(2) (1918), 366-374.

[2] Fishburn, P.C. and Trotter, W.T., Containment orders for similar ellipses with a common center. Discrete Mathematics 256 (2002), 129-136.

[3] Lefkaditis, G.E., Thomas, T.L. and Markatis, S., The four ellipses problem. International Journal of Geometry 5(2) (2016), 77-92.

[4] Müller, E. and Kruppa, E., Lehrbuch der Darstellenden Geometrie. Springer-Verlag, Wien, 1961.

[5] Peschka, G. A., Elementarer beweis des Pohlke'schen fundamentalsatzes der Axonometrie. Stzgsb. Math. Nat., Akad. Wien LXXVIII II Abth. (1879), 1043-1054.

[6] Sklenáriková, Z. and Pémová, M., The Pohlke-Schwarz theorem and its relevancy in the didactics of mathematics. Quaderni di Ricerca in Didattica. http://math.unipa.it/ grim/quad17_sklenarikova-pemova_07.pdf (2007).

\section{Affiliations}

THOMAS L. TOULIAS

AdDress: Avenue Charbo 20, Schaerbeek 1030, Brussels, Belgium.

E-MAIL: th.toulias@gmail.com

GEORGE E. LEFKADITIS

ADDRESS: University of Patras, Department of Architecture, Rio 26504, Patras, Greece.

E-MAIL: glef@upatras.gr 